\title{
Crecimiento económico e infraestructura de transportes y comunicaciones en el Perú
}

\author{
ROBERTO MACHADO \\ Hiroshi TOMA*
}

\begin{abstract}
RESUMEN
La presente investigación busca el aporte de la inversión pública en infraestructura de transportes y comunicaciones sobre el crecimiento económico de las regiones del Perú. Esto se realiza sobre la base de diversas estimaciones con datos de panel para las 24 regiones del Perú en el periodo 20042014. En primer lugar, para las estimaciones se usa una metodología convencional de datos de panel con efectos fijos. Se encuentra un aporte positivo de la inversión en infraestructura de transportes y comunicaciones sobre el PBI y el PBI por trabajador de las regiones. Luego, se sigue una metodología espacial de datos de panel, la cual toma en cuenta los efectos directos de la inversión en infraestructura dentro de una región, así como los efectos indirectos que ocurren entre regiones. Los resultados sugieren que la inversión en transportes afecta positivamente el PBI regional de forma directa, mientras que la inversión en comunicaciones aporta al PBI de manera indirecta.

Palabras clave: crecimiento económico regional, econometría espacial, inversión pública, transportes, comunicaciones
\end{abstract}

Código JEL: O47, R53

\section{Economic growth and transport and communications infrastructure in Peru}

\begin{abstract}
This research aims to measure the contribution of public investment in transport and communications infrastructure to economic growth in the regions of Peru. This is done on the basis of different estimations using panel data for the 24 regions of Peru, over the period 20042014. First, a conventional fixed-effect panel data method is used for the estimations, whereby investment in transport and communications infrastructure is found to have a positive effect on GDP and GDP per worker in the regions. Then, a spatial panel data methodology is followed, which takes into account the direct effects of investment in infrastructure within a region, as well as the indirect effects that occur between regions. Here, the results suggest that investment in transportation has a direct positive effect on regional GDP, while investment in communications contributes to GDP indirectly.
\end{abstract}

Keywords: Regional economic growth, spacial econometrics, transportation, communications JEL Code: O47, R5

* Contacto: roberto.machado.g@gmail.com; jtoma@pucp.pe. 


\section{INTRODUCCIÓN}

Indudablemente, la cobertura y la calidad de la infraestructura física y los servicios de transportes y comunicaciones son ingredientes importantes para el crecimiento económico. Desde el punto de vista analítico, Servén (2015) señala que la oferta de infraestructura puede afectar el crecimiento como un insumo productivo adicional, similarmente al trabajo, el capital humano y el capital físico distinto al de infraestructura; reduciendo el costo de otros factores e insumos intermedios (por ejemplo, bajando el costo de instalar nuevas empresas o de acceder a la educación y a la salud); y a través de externalidades positivas (efectos de derrame) sobre la productividad total de factores (PTF). Almeida y Guimarậes (2014) añaden otros canales mediante los cuales la infraestructura puede influenciar el crecimiento, tales como la mayor productividad laboral, la reducción de costos de transacción que facilita el acceso a productos y tecnología, y la mayor conectividad física que desarrolla los mercados regionales y fortalece los flujos de información.

Sobre este último punto, Webb (2013) encuentra que la conectividad de las áreas rurales en el Perú — medida como la inversa del tiempo que toma llegar desde el centro poblado donde vive un hogar hasta la capital del distrito - ejerce un efecto significativo sobre la productividad laboral. Por supuesto, este tiempo será menor, cuanto mejor sea la cobertura y calidad de la infraestructura de transportes.

En términos empíricos, Servén (2015) destaca dos aproximaciones usualmente utilizadas con datos macroeconómicos para evaluar el impacto de la infraestructura sobre el crecimiento. En primer lugar, el tratamiento de la infraestructura como otro insumo en la función de producción agregada de la economía, donde se estiman los efectos directos sobre el crecimiento, pero no los indirectos, asociados al impacto de los cambios en la infraestructura sobre la productividad de los otros insumos productivos. En segundo lugar, la estimación de regresiones de crecimiento con indicadores de infraestructura como variables explicativas, junto con otras variables de control usualmente encontradas en la literatura, donde se estiman los efectos totales (directos e indirectos). La mayoría de estos estudios han encontrado importantes efectos positivos de la infraestructura sobre el crecimiento, en especial en países en desarrollo, y cuando se usan indicadores físicos de infraestructura (por ejemplo, kilómetros de carreteras en el caso de transportes). Quizás las principales contribuciones a la comprensión de la relación empírica entre infraestructura y crecimiento en el Perú son los estudios de Vásquez y Bendezú (2008) y de Urrunaga y Aparicio (2012). Ambos confirman la importancia de este aspecto en el crecimiento, el primero respecto de la infraestructura vial, y el segundo en cuanto a las infraestructuras de energía, transportes y telecomunicaciones.

La fase de notable desempeño del crecimiento de la economía peruana, registrado durante el llamado "súper-ciclo" de los precios internacionales de las materias primas de 2003-2013 parece haber llegado a su fin. En efecto, mientras que el PBI se expandió a 
la tasa anual promedio de $6,4 \%$ en ese periodo (a precios constantes de 2007), el crecimiento se desaceleró perceptiblemente, registrando una tasa de $2,4 \%$ en 2014 . Entre enero y setiembre, la economía creció $2,6 \%$ con relación al mismo periodo de 2014 , mientras que distintas proyecciones ubican la tasa de crecimiento por debajo de 3\% para todo el año.

En este escenario, las insuficiencias en cantidad y calidad de la infraestructura física debieran ser materia de preocupación en el país. De hecho, estimaciones realizadas recientemente anuncian apreciables brechas de infraestructura física a nivel nacional, del orden US\$160.000 millones para el decenio 2016-2025 a precios constantes de 2015 (AFIN, 2015). En consecuencia, se requeriría un importante esfuerzo de inversión en infraestructura durante los próximos años, a fin de apuntalar el crecimiento económico y mejorar la calidad de vida de la ciudadanía.

Este estudio pretende hacer una contribución a la comprensión del rol de la infraestructura en el crecimiento económico del Perú, con énfasis en la de transportes y comunicaciones sobre la base de una estimación econométrica con datos de panel para las 24 regiones del Perú en el periodo 2004-2014. En la siguiente sección se discute brevemente las brechas de infraestructura que enfrenta el país en la actualidad. La tercera sección pasa revista a la literatura teórica y empírica sobre el tema, mientras que la cuarta analiza la evidencia en el caso peruano. En la quinta sección se presenta la parte sustantiva de este trabajo: la metodología empírica, los datos utilizados y los resultados econométricos. Destaca la utilización de dos metodologías complementarias. En primer lugar, la estimación tradicional con métodos de datos de panel de efectos fijos, que permite estimar las elasticidades inversión-producto tanto para la infraestructura de transportes como para la de comunicaciones. En segundo lugar, la estimación espacial, que permite explorar el impacto de la inversión en transportes o en comunicaciones sobre el producto de la misma región (efecto directo). Más importante, esta metodología permite estimar también el efecto promedio de la inversión en todas las 23 otras regiones sobre el producto de la región restante (efecto indirecto). Las implicancias de política para la inversión en transportes y comunicaciones derivadas de la estimación espacial son poderosas, como se verá más adelante. Finalmente, la sexta sección presenta las conclusiones.

\section{BRECHAS DE INFRAESTRUCTURA FÍSICA EN EL PERÚ}

La brecha de infraestructura física se puede entender como la inversión que falta ejecutar en un determinado país en materia de infraestructura para que se cumpla con dos objetivos: que el país alcance el nivel del stock de infraestructura con el que cuentan otros países de características socioeconómicas similares, y que se pueda responder satisfactoriamente a la creciente demanda por infraestructura física (AFIN, 2015). Al déficit en el primer aspecto se le denomina brecha horizontal, y a la segunda brecha vertical. 
A precios constantes de 2015, AFIN (2015) estimó la brecha en infraestructura en US\$68.800 millones para el periodo 2016-2020, y en US\$90 734 millones para el periodo 2021-2025. En total, el déficit de infraestructura entre 2016 y 2025 ascendería a unos US\$160 .000 millones, 83\% del PBI de 2015 (US\$193 000 millones) ${ }^{1}$.

En el Cuadro 1 se presentan las estimaciones de las brechas de infraestructura para el periodo 2016-2025, dividido en dos sub-periodos. En el mediano plazo (de 2016 a 2020), el sector que demandaría mayor inversión es el de transporte (30,9\% del total), principalmente en carreteras $(16,3 \%)$ y ferrocarriles $(11,1 \%)$, con puertos y aeropuertos requiriendo mucho menos recursos (1,5\% y 2,1\% del total, respectivamente). Le sigue en importancia el sector de comunicaciones (18,3\% del total), con una mayor participación del acceso a banda ancha (14,6\% del total). En el periodo 2016-2025, la brecha de infraestructura sería mayor nuevamente en transporte $(39,9 \%$ del total), principalmente en carreteras $(22,8 \%)$ y ferrocarriles $(10,3 \%)$. Aunque el segundo lugar en términos de requerimientos de inversión en este subperiodo correspondería a energía $(21,9 \%)$, el déficit de infraestructura también es importante en telecomunicaciones (15,9\%), sobre todo en banda ancha (11,1\%). En el periodo completo 2016-2025, los mayores requerimientos de inversión en infraestructura corresponderían a transportes, con más de la tercera parte del total (36\%), destacando las brechas de carreteras $(20 \%)$ y ferrocarriles $(10,6 \%)$. El déficit de infraestructura de telecomunicaciones, por su parte, daría cuenta de $16,9 \%$ del total, en especial el relacionado al acceso a banda ancha $(12,6 \%)$.

Cuadro 1. Brecha de infraestructura, 2016-2025

\begin{tabular}{lcccccc}
\hline & $\begin{array}{c}\text { Mediano plazo } \\
\text { 2016-2020 }\end{array}$ & Porcentaje & $\begin{array}{c}\text { Periodo } \\
\text { 2021-2025 }\end{array}$ & Porcentaje & $\begin{array}{c}\text { Largo plazo } \\
\text { 2016-2025 }\end{array}$ & Porcentaje \\
\hline Telecomunicaciones & 12603 & 18,3 & 14432 & 15,9 & 27036 & 16,9 \\
- Telefonía móvil & 2552 & 3,7 & 4362 & 4,8 & 6884 & 4,3 \\
- Banda ancha & 10081 & 14,6 & 10070 & 11,1 & 20151 & 12,6 \\
\hline Agua y saneamiento & 6970 & 10,1 & 5282 & 5,8 & 12252 & 7,7 \\
- Agua potable & 1624 & 2,4 & 1004 & 1,1 & 2629 & 1,6 \\
- Saneamiento & 5345 & 7,8 & 4278 & 4,7 & 9623 & 6,0 \\
\hline Infraestructura hidráulica & 4537 & 6,6 & 3940 & 4,3 & 8477 & 5,3 \\
\hline Transporte & 21253 & 30,9 & 32246 & 39,9 & 57499 & 36,0 \\
- Ferrocarriles & 7613 & 11,1 & 9370 & 10,3 & 16983 & 10,6 \\
- Carreteras & 11184 & 16,3 & 20667 & 22,8 & 31850 & 20,0 \\
- Aeropuertos & 1419 & 2,1 & 959 & 1,1 & 2378 & 1,5 \\
- Puertos & 1037 & 1,5 & 5250 & 5,8 & 6287 & 3,9 \\
\hline
\end{tabular}

1 El PBI en dólares a precios corrientes en 2015 es similar al de 2012 debido a la elevación del tipo de cambio, que pasó de 2,64 a 3,20 soles por dólar entre esos mismos años. Con el tipo de cambio de 2012, el PBI de 2015 hubiera sido de US\$234 000 millones. 


\begin{tabular}{lcccccc}
\hline & $\begin{array}{c}\text { Mediano plazo } \\
\text { 2016-2020 }\end{array}$ & Porcentaje & $\begin{array}{c}\text { Periodo } \\
\text { 2021-2025 }\end{array}$ & Porcentaje & $\begin{array}{c}\text { Largo plazo } \\
\text { 2016-2025 }\end{array}$ & Porcentaje \\
\hline Energía & 11388 & 16,5 & 19387 & 21,4 & 30775 & 19,3 \\
\hline Salud & 9472 & 13,8 & 9472 & 10,4 & 18944 & 11,9 \\
\hline Educación & 2592 & 3,8 & 1976 & 2,2 & 4568 & 2,9 \\
- Inicial & 1037 & 1,5 & 585 & 0,6 & 1621 & 1,0 \\
- Primaria & 137 & 0,2 & 137 & 0,2 & 274 & 0,2 \\
- Secundaria & 1418 & 2,1 & 1254 & 1,4 & 2672 & 1,7 \\
\hline TOTAL & $\mathbf{6 8 8 1 5}$ & $\mathbf{1 0 0 , 0}$ & $\mathbf{9 0 7 3 4}$ & $\mathbf{1 0 0 , 0}$ & $\mathbf{1 5 9 5 4 9}$ & $\mathbf{1 0 0 , 0}$ \\
\hline
\end{tabular}

Fuente: AFIN (2015).

Llama la atención lo reducido de la brecha estimada en puertos y, especialmente, aeropuertos, que para el decenio 2016-2025 ascenderían a unos US\$6300 y US\$2400 millones, respectivamente (a precios constantes de 2015). Por ejemplo, solo la segunda pista de aterrizaje del Aeropuerto Jorge Chávez involucraría una inversión de US\$1200 millones, la mitad de lo estimado para el periodo completo. Del mismo modo, el concesionario del Aeropuerto de Chinchero ganó la buena pro con un costo de US\$265 millones. Ambas obras deberían ser ejecutadas a 2021. Más allá de que las brechas mostradas en el Cuadro 1 están expresadas a precios constantes de 2015, mientras que los montos de inversión indicados para los dos proyectos de inversión mencionados están expresados a precios corrientes, parecería que la brecha en infraestructura de aeropuertos está subestimada. Algo similar podría suceder con la brecha en infraestructura de puertos y otros sectores.

\section{TEORÍA Y EVIDENCIA SOBRE INFRAESTRUCTURA Y CRECIMIENTO}

Existe una amplia variedad de estudios sobre los efectos de la infraestructura física en el crecimiento económico tanto en términos teóricos como empíricos. Calderón y Servén (2014) realizan una revisión de esta literatura. Usualmente, el impacto de la infraestructura sobre el crecimiento se ha modelado incluyendo el stock de infraestructura o el flujo de servicios de infraestructura como otro insumo en la función de producción agregada de la economía, junto con el trabajo, el capital humano y el capital físico distinto de la infraestructura. Así, un aumento en el insumo de infraestructura incrementará el producto de manera directa, pero también indirecta, a través de sus efectos positivos sobre la productividad de los otros insumos productivos. Sin embargo, esta mayor infraestructura debe ser financiada vía un incremento de impuestos, lo que desincentiva el uso de otros insumos, con el consiguiente impacto negativo sobre el efecto indirecto vía la mayor productividad. 
Barro (1990) analiza este problema, en el marco de un modelo de crecimiento endógeno donde la contribución del gobierno al PBI se materializa a través del gasto público que provee servicios productivos como los de infraestructura (y no vía el stock de infraestructura) financiado con un impuesto proporcional a la renta. La maximización de la función de utilidad de la familia representativa, implica un nivel de gasto público productivo como porcentaje del PBI $(g / y)$ que debe ser igual a la elasticidad entre estas dos variables $\left(\frac{\partial y}{\partial g} \cdot \frac{g}{y}\right)$. En ese punto, no solo se maximiza el bienestar de la economía, sino también la tasa de crecimiento del producto, que es igual a la tasa de crecimiento del consumo y del stock de capital físico distinto a la infraestructura. En el caso en que el gasto público productivo (inversión en infraestructura) exceda ese nivel, la utilidad y la tasa de crecimiento de la economía caerán, debido a que las distorsiones generadas por la más alta tasa de impuesto a la renta necesaria para financiar ese mayor gasto desincentivará la inversión privada (la utilización de otros factores productivos tales como el capital humano y el capital físico distinto de la infraestructura), y con ella la tasa de crecimiento de la economía. De manera contraria, si el gasto público productivo es demasiado bajo, el impacto positivo que ejerce sobre la productividad del capital privado será insuficiente, lo que también redundará en una menor inversión privada y una menor tasa de crecimiento. Así, en este marco, la inversión pública en infraestructura afecta la tasa de crecimiento de largo plazo (estado estacionario). Sería interesante extender este modelo considerando modos de financiamiento de la inversión pública alternativos al impuesto a la renta. En particular, introduciendo la posibilidad de participación del sector privado a través de asociaciones público-privadas (APP).

Utilizando un modelo de crecimiento endógeno similar al de Barro (1990), pero incluyendo el capital público en la función de producción, Futagami et al. (1993), encuentran que la inversión pública como porcentaje del PBI $(i / y)$ que maximiza la tasa de crecimiento económico sigue siendo aquella donde se iguala con la elasticidad correspondiente $\left(=\frac{\partial y}{\partial i} \cdot \frac{i}{y}\right)$. Sin embargo, en este punto la función de utilidad de la familia representativa es menor, con lo que para maximizar el bienestar se debe reducir ily. Calderón y Servén (2014) racionalizan este resultado señalando que la inversión pública toma tiempo en devenir productiva, de modo que este rezago impone sacrificios adicionales de consumo en el presente para obtener consumo en el futuro.

Como se señaló en la primera sección, al introducir la infraestructura como otro insumo en la función de producción se puede derivar el impacto directo de esta sobre la tasa de crecimiento. Sin embargo, la infraestructura también puede entrar en la función de producción de manera indirecta a través de su efecto sobre la productividad total de factores (PTF). Más aún, la infraestructura física — sobre todo de transportes y de comunicaciones - también puede apoyar la acumulación de otros insumos productivos como el capital físico y el capital humano. 
Dos aspectos adicionales importantes en el abordaje analítico del efecto de la infraestructura sobre el crecimiento, destacados por Calderón y Servén (2014) son el de congestión y el de efectos de red. En el primer caso, muchos tipos de infraestructura, incluyendo la de transportes y comunicaciones, están sujetos a rivalidad en el consumo y son pasibles de exclusión en su uso. Es el caso, por ejemplo, de las carreteras saturadas por el uso masivo simultáneo, y que pueden financiarse con la imposición de peajes. Así, se habla de congestión absoluta cuando la cantidad de servicios de infraestructura consumidos por un productor individual depende negativamente del uso agregado, mientras que la congestión relativa supone que esta cantidad depende negativamente del ratio uso individual/uso agregado. No obstante, incluso en presencia de congestión, los resultados discutidos anteriormente en el marco de un modelo de crecimiento endógeno se mantienen: con la infraestructura modelada como flujo de servicios productivos, el nivel de $g / y$ que maximiza el bienestar (y la tasa de crecimiento) es el que se iguala con la elasticidad entre ambas variables; si se modela como stock de capital público, ese nivel de $g / y$ maximizará la tasa de crecimiento, pero será excesivo para la maximización del bienestar.

De otro lado, en presencia de efectos de red asociados a la infraestructura, se puede generar significativas no linealidades en su productividad marginal. Por ejemplo, la construcción de una carretera (o la instalación de una antena de teléfonos celulares) puede tener un efecto muy limitado si no hay una red vial desarrollada (o si no hay una red de antenas instalada). Adicionalmente, esas no linealidades pueden dar lugar a equilibrios múltiples y a trampas de pobreza, donde un entorno pobre en infraestructura implica una baja productividad marginal de esta, lo que puede llevar a la economía a un equilibrio "malo" de bajo ingreso (o crecimiento). Sin embargo, en la tradición de Rosentein-Rodan (1943), un gran empujón (big push) —en el sentido de un gran esfuerzo de inversión en infraestructura - puede hacer que la economía se desplace a un equilibrio "bueno" de alto ingreso (o crecimiento).

En cuanto a la evidencia empírica sobre la relación entre crecimiento e infraestructura, tal vez Straub (2011) realiza la contribución más importante en términos de la revisión de los resultados de diversos estudios. Su análisis se basa en la siguiente función de producción general:

(1) $Y=A\left(\xi, K_{I}\right) \cdot F\left(K, L, I\left(K_{I}\right)\right)$

donde $Y$ es el producto agregado, $A$ es el factor de productividad, $K$ es el stock de capital distinto a la infraestructura, $K_{I}$ es el stock de capital de infraestructura, $L$ representa las horas trabajadas por la fuerza laboral, e $I\left(K_{I}\right)$ es una variable de insumos intermedios. Así, aumentos en $K_{I}$ disminuye los costos de los insumos intermedios contenidos en la función de producción, $F($.). El factor de producción depende de dos términos: cambios en $A($.) pueden deberse a externalidades positivas derivadas de la acumulación de capital de infraestructura $\left(K_{I}\right)$, y a las demás externalidades positivas provenientes de otras fuentes $(\xi)$. 
A los efectos de la infraestructura a través de $I($.), Straub (2011) los llama "efectos directos", mientras que a los que operan a través de $A($.$) , los llama "efectos indirectos".$

¿Por qué se incluye $K_{I}$ en $F($.$) a través de I($.) en lugar de directamente como otro factor de producción? La introducción del capital de infraestructura directamente en la función de producción asume que la infraestructura es un bien público puro, es decir, que está sujeto a las condiciones de no rivalidad y de no exclusión en el consumo. Sin embargo, esto es cierto solo parcialmente, puesto que la infraestructura es crecientemente intermediada por el mercado (a través del pago de peajes y tarifas eléctricas, por ejemplo) y por tanto tiene características de bienes privados. De modo que su incorporación en $F($.$) vía I($.$) como factor de producción de ciertos servicios productivos que$ entran en la función de producción agregada parece más adecuado.

¿Por qué se incluye $K_{I}$ en $A($.)? En contraposición a lo mencionado en el párrafo anterior, a pesar de que la infraestructura es crecientemente intermediada por el mercado, usualmente sus precios no reflejan su productividad marginal. Si el costo unitario de la infraestructura no está determinado por el mercado, entonces no conviene introducirla como otro factor de producción en $F($.) puesto que las firmas privadas no podrían tomar decisiones informadas sobre la cantidad de infraestructura que utilizan. Por esta razón, se puede considerar que la infraestructura es parte del término de la PTF, $A$, porque reduce los costos de producción o a través de economías de escala asociadas a la expansión de los mercados.

A partir de este marco de análisis, Straub (2011) analiza las respuestas que la literatura empírica ha encontrado a algunas preguntas relevantes, que divide en tres:

i) La magnitud y naturaleza de los efectos de la infraestructura sobre el producto y el crecimiento.

a) Comparación de las elasticidades de $K$ y $K_{I}$ respecto del producto.

b) Si es posible distinguir los efectos directos (vía $I($.$) ) de los indirectos (vía A($.$) ).$

c) $\mathrm{Si}$ es que es posible estimar los efectos indirectos, ver cómo se comparan las contribuciones de las externalidades de infraestructura (capturadas por $K_{I}$ en $A()$.$) con las otras (capturadas por \xi$ en $A($.$) ).$

ii) Si el efecto de la inversión adicional en infraestructura es permanente o transitorio.

iii) Si se puede calcular un stock de capital en infraestructura óptimo.

La segunda y la tercera preguntas merecen cierta elaboración. La segunda interrogante se reduce a si un aumento de la infraestructura es capaz de generar suficientes externalidades como para impedir que el stock de capital a nivel agregado (en infraestructura y en otro tipo de capital) presente retornos decrecientes, de modo que lleve a una dinámica de crecimiento endógeno. Eliminando $\xi$ para simplificar, e introduciendo $K_{I}$ como otro factor de producción en una formulación Cobb-Douglas de la ecuación (1): 
(2) $Y=\left(A \cdot K_{I}^{\phi}\right) \cdot K^{\eta} \cdot K_{I}^{\gamma} \cdot L^{1-\eta-\gamma}$

donde $A \cdot K_{I}^{\phi}$ es el término de productividad y $\phi$ su elasticidad con respecto al capital de infraestructura. En este marco, incluso si se supone recursos decrecientes a escala en los factores directamente reproducibles $\left(K_{I}\right.$ y $\left.K\right)$, es decir, $\eta+\gamma<1$, las externalidades de la infraestructura podrían generar retornos constantes a escala, de modo que $\eta+\gamma+\phi=1$.

La tasa de crecimiento del producto agregado será igual a la suma ponderada de las tasas de crecimiento del capital general y del capital de infraestructura: $\hat{q}=\phi \cdot \hat{k}_{I}+$ $\eta \cdot \hat{k}+\gamma \cdot \hat{k}_{I}$ (dado A), donde las letras minúsculas representan las correspondientes mayúsculas por trabajador, y el símbolo “ $\wedge$ ” indica tasa de crecimiento porcentual. En este contexto, cualquier cambio (de una sola vez) que genere un aumento en las tasas de crecimiento de estos dos tipos de capital tendrá un efecto permanente sobre la tasa de crecimiento de la economía. Por el contrario, si $\eta+\gamma+\phi<1$, la tasa de crecimiento regresará a su nivel inicial.

Respecto de la tercera pregunta referente a la determinación de un stock de infraestructura óptimo, se requeriría igualar la productividad marginal (social, dada la existencia de una externalidad positiva asociada a la acumulación de $K_{I}$ ) con el costo marginal del capital de infraestructura. Reformulando (1) como:

(3) $Y=A \cdot K^{\eta} \cdot K_{I}^{\gamma} \cdot L^{1-\eta-\gamma}$

y suponiendo como Barro (1990) que la inversión pública en infraestructura $(g)$ es una fracción constante del producto $(y), \tau=g / y$, se puede mostrar que el $g / y$ que maximiza el bienestar y la tasa de crecimiento del producto, del consumo y del stock de capital distinto a infraestructura (todo en términos per cápita), es $(g / y)^{*}=\tau^{*}=\gamma / \eta+\gamma$. A pesar de la importancia analítica de la formulación de Barro (1990) al incluir el gasto público en infraestructura en un modelo de crecimiento endógeno (y su financiamiento con un impuesto a la renta proporcional, cuya tasa es $\tau$ ), citando a Pritchett (2000), Straub (2011) destaca que esto no sería relevante empíricamente, dado que los gobiernos no se comportan como optimizadores y a la dificultad de mapear la inversión pública con la infraestructura física existente debido a ineficiencia, corrupción y uso electoral ("pork barrel”) de la inversión pública en infraestructura.

A partir del marco analítico descrito, Straub (2011) procede a evaluar la evidencia empírica respecto de la elasticidad-producto del capital de infraestructura (pregunta (i) (a)). Encuentra que de un total de 77 estudios revisados, 43 (56\%) encuentran un efecto positivo y estadísticamente significativo de la infraestructura sobre el crecimiento, $29(38 \%)$ no encuentran ninguna relación estadísticamente distinta de $0, y$ solo $5(7 \%)$ hallan un efecto negativo y estadísticamente significativo. El Cuadro 2 muestra los resultados. Se observa que de los 34 estudios que utilizan el capital físico como variable de infraestructura - es decir, la acumulación de la inversión o el gasto público en infraestructura-, el $41 \%$ encuentra un efecto positivo sobre el crecimiento, 
$44 \%$ ningún efecto, y $15 \%$ un impacto negativo. De los cuatro estudios que emplean el capital físico en transporte como variable de infraestructura, tres encuentran un efecto positivo sobre el crecimiento. Lo mismo sucede con los dos estudios que consideran el capital físico en telecomunicaciones como variable de infraestructura.

Cuadro 2. Efecto estimado de la infraestructura sobre el crecimiento (Porcentaje del total de estudios revisados)

\begin{tabular}{lccc}
\hline Variable de infraestructura & $\begin{array}{c}\text { Negativo y estadísticamente } \\
\text { significativo }\end{array}$ & Ninguno & $\begin{array}{c}\text { Positivo y estadísticamente } \\
\text { significativo }\end{array}$ \\
\hline Capital público (34) & 14,7 & 44,1 & 41,2 \\
- Agregado (27) & 18,5 & 48,2 & 33,3 \\
- Transporte (4) & 0 & 25,0 & 75,0 \\
- Telecomunicaciones (2) & 0 & 0 & 100,0 \\
- Agua (1) & 0 & 100,0 & 0 \\
\hline Indicador físico (43) & 0 & 32,6 & 67,4 \\
- Sintético (6) & 0 & 16,7 & 83,3 \\
- Electricidad (11) & 0 & 44,4 & 54,6 \\
- Caminos (10) & 0 & 40,0 & 60,0 \\
- Telecomunicaciones (14) & 0 & 21,4 & 78,6 \\
- Agua (1) & 0 & 0 & 100,0 \\
- Saneamiento (1) & 0 & 100,0 & 0 \\
\hline TOTAL (77) & 6,5 & 37,7 & 55,8 \\
\hline
\end{tabular}

Fuente: Straub (2011).

Nota: Entre paréntesis aparece el número de estudios que incluye cada tipo de variable explicativa.

La influencia positiva de la infraestructura sobre el crecimiento económico es más nítida cuando se usan indicadores físicos como variable de infraestructura, en tanto ninguno de los 43 estudios revisados por Straub (2011) encuentra un impacto negativo, mientras que dos de cada tres (67\%), hallan un impacto positivo. De los diez que emplean alguna variable de transportes (caminos), el 60\% encuentran un efecto positivo, resultado similar al encontrado en cerca de cuatro de cada cinco (79\%) de los catorce que utilizan una variable física de telecomunicaciones.

En cuanto a la magnitud del impacto estimado de la infraestructura sobre el crecimiento, Urrunaga y Aparicio (2012) elaboran un cuadro resumen de los principales estudios que estiman esta elasticidad a través de regresiones econométricas hasta inicios de la presente década. El Cuadro 3 lo reproduce. La discusión sobre la evidencia empírica del caso peruano se realiza en la siguiente sección. Puede observarse que cuando se emplea una variable de gasto público como indicador de infraestructura, las elasticidades estimadas toman valores más extremos, yendo de 0,39 para el gasto público en capital excluyendo la defensa nacional en el estudio de Aschauer (1989) para los Estados Unidos en 1949-1985, a -0,058 para el gasto público en agua y desagüe 
en García Milà et al. (1996) para un panel de 48 estados del mismo país. Los valores de las elasticidades estimadas se ubican en un rango más acotado cuando se usan indicadores físicos como variables de infraestructura. En este caso, van de 0,156 para la energía en el estudio de Esfahani y Ramírez (2003) para un panel de 75 países en 1965-1995, y -0,043 para los caminos en Straub et al. (2008) para un panel de 40 países de bajos ingresos. En conclusión, existe una gran variedad de valores estimados para las elasticidades que miden el impacto de variaciones en la infraestructura sobre el crecimiento.

Cuadro 3. Evidencia empírica sobre el efecto de la infraestructura física sobre el crecimiento económico a partir de regresiones econométricas

\begin{tabular}{|c|c|c|c|c|}
\hline Estudio & País(es) & Muestra & Variable de infraestructura & Elasticidad estimada \\
\hline Aschauer (1989) & Estados Unidos & $1949-1985$ & $\begin{array}{l}\text { Gasto público en capital } \\
\text { no militar }\end{array}$ & 0,39 \\
\hline Munell (1990) & Estados Unidos & $1947-1988$ & Gasto público en infraestructura & 0,34 \\
\hline $\begin{array}{c}\text { Easterly y Rebelo } \\
\text { (1993) }\end{array}$ & $\begin{array}{l}28 \text { países en } \\
\text { desarrollo }\end{array}$ & $1970-1988$ & $\begin{array}{c}\text { Gasto público en transportes } \\
\text { y telecomunicaciones }\end{array}$ & 0,16 \\
\hline $\begin{array}{c}\text { Devarajan et al. } \\
(1996)\end{array}$ & $\begin{array}{l}43 \text { países en } \\
\text { desarrollo }\end{array}$ & $1970-1990$ & $\begin{array}{c}\text { Gasto público en transportes } \\
\text { y comunicaciones }\end{array}$ & $-0,025$ \\
\hline $\begin{array}{l}\text { García-Milà } \\
\text { et al. (1996) }\end{array}$ & $\begin{array}{l}\text { Estados Unidos } \\
\text { (48 estados) }\end{array}$ & $1971-1983$ & $\begin{array}{c}\text { Gasto público en agua y desagüe, } \\
\text { y autopistas }\end{array}$ & $\begin{array}{c}-0,058 \\
y-0,029\end{array}$ \\
\hline $\begin{array}{c}\text { Sánchez-Robles } \\
\text { (1998) }\end{array}$ & $\begin{array}{c}57 \text { países } \\
17 \text { países de } \\
\text { América Latina }\end{array}$ & $1970-1985$ & Índice de infraestructura & $\begin{array}{l}0,009 \\
0,012\end{array}$ \\
\hline $\begin{array}{l}\text { Duggal et al. } \\
\quad(1999)\end{array}$ & Estados Unidos & 1960-1989 & $\begin{array}{c}\text { Gasto público en caminos } \\
\text { y estructuras }\end{array}$ & 0,27 \\
\hline Canning (1999) & $\begin{array}{l}57 \text { países en } \\
\text { desarrollo }\end{array}$ & $1960-1990$ & $\begin{array}{l}\text { Indicador físico de } \\
\text { telecomunicaciones }\end{array}$ & 0,139 \\
\hline $\begin{array}{c}\text { Esfahani y } \\
\text { Ramírez (2003) } \\
\end{array}$ & 75 países & $1965-1995$ & $\begin{array}{l}\text { Indicadores físicos de } \\
\text { telecomunicaciones y energía }\end{array}$ & 0,091 у 0,156 \\
\hline $\begin{array}{c}\text { Calderón y } \\
\text { Servén (2004) }\end{array}$ & 101 países & $1960-2000$ & $\begin{array}{l}\text { Índice de infraestructura } \\
\text { física }\end{array}$ & $\begin{array}{c}\text { 0,0195 y 0,0207, } \\
\text { dependiendo del } \\
\text { método de estimación }\end{array}$ \\
\hline $\begin{array}{c}\text { Rivera y Toledo } \\
(2004)\end{array}$ & Chile & $1975-2000$ & $\begin{array}{c}\text { Inversión pública sectorial } \\
\text { en infraestructura }\end{array}$ & 0,16 \\
\hline $\begin{array}{l}\text { Straub et al. } \\
\quad(2008)\end{array}$ & $\begin{array}{l}92 \text { países } \\
\text { emergentes } \\
40 \text { países de } \\
\text { bajos ingresos }\end{array}$ & $1971-1995$ & $\begin{array}{c}\text { Indicadores físicos } \\
\text { de infraestructura de } \\
\text { telecomunicaciones, caminos } \\
\text { y estructuras }\end{array}$ & $\begin{array}{l}0,028 ; 0,029 \text { y } 0,018 \\
0,03 ;-0,043 \text { y } 0,028\end{array}$ \\
\hline
\end{tabular}

Fuente: Urrunaga y Aparicio (2012).

Respecto de los efectos indirectos de la infraestructura sobre el crecimiento (preguntas (i) (b) y (i) (c)), Straub (2011) encuentra pocos estudios que abordan esta problemática. 
El primer problema es que cuando se utiliza una función de producción Cobb-Douglas (como en los estudios de contabilidad del crecimiento), hay muy poco que decir acerca de los efectos indirectos de la infraestructura, porque esta formulación no permite distinguirlos de los efectos directos (separar $\gamma$ y $\phi$ en (2)). En contabilidad del crecimiento sucede algo similar, dado que como el capital de infraestructura tiene un carácter semipúblico (parte es intermediado por el mercado), no se le remunera según su productividad marginal. En consecuencia, su participación en el producto solo puede adivinarse, lo que implica que las estimaciones deben ser tomadas con cautela. Aunque evidencia más reciente sugiere que los efectos indirectos de la infraestructura pueden ser importantes, no existe aún una metodología definitiva para evaluarlos empíricamente.

Con relación al carácter temporal o permanente de los efectos de la infraestructura (pregunta (ii)), Straub (2011) señala que las especificaciones que utilizan el nivel de producto como variable dependiente en lugar de la tasa de crecimiento del producto o la productividad tienden a respaldar más el efecto positivo de la infraestructura. Esto podría indicar que en la mayoría de los casos el efecto de la inversión en infraestructura sobre la tasa de crecimiento es temporal y no permanente (afecta el nivel del producto, pero no su tasa de crecimiento).

Sin embargo, a partir de sus estimaciones, Calderón y Servén (2004) señalan que si las cantidades y calidades de los stocks de infraestructura de América Latina fueran del nivel de los de los países del Este Asiático, la tasa de crecimiento de largo plazo del PBI sería 3\% mayor. No obstante, Straub (2011) es escéptico sobre esta interpretación, argumentando que si fuera cierta, la magnitud del efecto causal del capital de infraestructura sobre la tasa de crecimiento, todo el crecimiento de largo plazo de Estados Unidos y de Europa (2\%-3\% anual en el largo plazo) podría atribuirse a sus mayor disponibilidad de infraestructura física, tanto en cantidad como en calidad. Alternativamente, Straub (2011) interpreta los resultados de Calderón y Servén (2004) como sobreestimados, debido a que estarían incorporando no solo el impacto directo e indirecto de $K_{I}$ sobre la tasa de crecimiento, sino también parte de las externalidades generadas por otras causas ( $\xi$ en la ecuación (1)). Asimismo, se podría interpretar estos resultados como que con un gran esfuerzo de inversión en infraestructura, los países de América Latina podrían experimentar mayores tasas de crecimiento por un periodo de tiempo prolongado. Es decir, una argumentación similar a la del gran empujón para saltar de un equilibrio de bajos ingresos hacia uno de altos ingresos. Esto es muy distinto a decir que mayores stocks de infraestructura llevan a más altas tasas de crecimiento de estado estacionario en estos países; aunque efectos transitorios por un periodo prolongado podrían parecer efectos permanentes.

Finalmente, a pesar de la gran relevancia de la determinación del stock de capital de infraestructura óptimo (pregunta (iii)), la literatura le ha prestado muy poca atención, presumiblemente debido a las dificultades metodológicas que entraña un cálculo de este tipo. Siguiendo a Gramlich (1994), Straub (2011) indica que se han empleado varios métodos para tratar de estimar el stock de infraestructura óptimo, incluyendo evaluaciones 
acerca de las necesidades, mediciones políticas basadas en el comportamiento de los votantes, mediciones de la tasa de retorno, y estimaciones econométricas. Sin embargo, ninguno ha resultado en respuestas y metodologías concluyentes.

\section{INFRAESTRUCTURA Y CRECIMIENTO: EVIDENCIA PARA EL PERÚ}

Como se mencionó en la primera sección, los estudios más importantes sobre el impacto de la infraestructura sobre el crecimiento en el Perú son los de Vásquez y Bendezú (2008) y Urrunaga y Aparicio (2012). El primero es un análisis integral de la relación entre la infraestructura y el crecimiento desde diversas perspectivas y aproximaciones econométricas. Sus resultados indican que - en un contexto de análisis de series de tiempo y cointegración - el efecto agregado de la expansión de la infraestructura vial sobre el crecimiento agregado es positivo inicialmente, pero tiende a desaparecer en el tiempo. Esto está en línea con la interpretación de los resultados de Calderón y Servén (2004) para los países de América Latina —en un contexto de análisis dinámico de datos de panel y estimación por el Método Generalizado de Momentos (MGM) — sugerida por Straub (2011) y comentada en la sección anterior, en el sentido de que el impacto de la infraestructura sobre el crecimiento sería transitorio, es decir, que aumentaría el nivel del producto, pero no su tasa de crecimiento de largo plazo. Más importante, en el contexto de una estimación con datos de panel para las regiones del país, Vásquez y Bendezú (2008) hallan que el aumento en el nivel de la infraestructura de energía, transportes y telecomunicaciones, también impactan positivamente en el crecimiento de las regiones, y explican parte de las desigualdades en el nivel de ingreso regional. Finalmente, desde una perspectiva distinta, este estudio también evalúa los efectos secundarios que genera la construcción de caminos vía la integración de mercados. En particular, realiza un análisis de integración espacial para tres productos de exportación: espárragos, café y polos de algodón. De acuerdo a los resultados, la infraestructura vial aumenta la eficiencia de los mercados al acelerar el ajuste de precios ante una perturbación exógena, en especial en mercados agrícolas como el del espárrago.

El estudio de Urrunaga y Aparicio (2012), por su parte, realiza una estimación econométrica con datos de panel para las 24 regiones del país, bajo distintas especificaciones y utilizando diversos estimadores. Sus resultados confirman que las infraestructuras de servicios públicos (carreteras, electricidad y telecomunicaciones) son relevantes para explicar las diferencias en el PBI regional por habitante y que hay diferencias significativas en los efectos relativos que los tres tipos de infraestructura ejercen sobre el PBI per cápita de cada región.

En el Cuadro 4 se presentan los resultados de ambos estudios respecto del impacto de la infraestructura sobre el crecimiento de las 23 o 24 regiones del país ${ }^{2}$ utilizando técnicas

2 Como la región Ucayali fue creada a mediados de los años 1980, Vásquez y Bendezú (2008) agregaron los datos de esta región con los de Loreto, de modo que trabajan con 23 regiones. 
de estimación con variables instrumentales para datos de panel dinámico de Arellano y Bond (1991). Lo primero que salta a la vista es que mientras Vásquez y Bendezú (2008) estiman un modelo donde la variable dependiente es la tasa de crecimiento del PBI, en el utilizado por Urrunaga y Aparicio (2012) la variable explicada es la tasa de crecimiento del PBI per cápita. Asimismo, puede observarse que, aparte de la tasa de crecimiento del PBI rezagada un periodo $\left(\Delta \operatorname{Ln}(\mathrm{PBI})_{t-1}\right)$ que aparece en los dos estudios como variable dependiente - PBI total en Vásquez y Bendezú (2008), PBI per cápita en Urrunaga y Aparicio (2012)—, también consideran como variables explicativas indicadores de infraestructura de tres tipos (electricidad, telecomunicaciones y transportes), el stock de capital privado, y la PEA educada (es decir, con educación secundaria o superior). Sin embargo, el primer estudio incluye las variables explicativas en niveles, mientras que el segundo incluye las tasas de crecimiento de las mismas. Vásquez y Bendezú (2008) también incluyen la superficie agrícola como variable de control.

Cuadro 4. Impacto de la infraestructura sobre el crecimiento de las regiones del Perú (Método de estimación: Método Generalizado de Momentos ${ }^{a}$ )

Vásquez y Bendezú (2008)

\begin{tabular}{|c|c|c|}
\hline \multicolumn{3}{|c|}{ Vásquez y Bendezú (2008) } \\
\hline \multicolumn{3}{|c|}{ Variable dependiente: Tasa de crecimiento del PBI } \\
\hline Variable explicativa & Descripción & $\begin{array}{c}\text { Parámetro } \\
\text { estimado }\end{array}$ \\
\hline Constante & Toma el valor de 1 en toda la muestra & $-0,085^{* * *}$ \\
\hline$\Delta \operatorname{Ln}(\mathrm{PBI})_{t-1}$ & $\begin{array}{l}\text { Tasa de crecimiento del PBI en soles a precios de } 1994 \\
\text { rezagado un periodo }\end{array}$ & $-0,066^{* * *}$ \\
\hline Ln(Indicador de electricidad) & Ln de la potencia eléctrica instalada en megawatts/hora & $0,114^{* * *}$ \\
\hline $\begin{array}{l}\text { Ln(Indicador de } \\
\text { telecomunicaciones) }\end{array}$ & Ln del número de líneas telefónicas en servicio & $0,051^{* * *}$ \\
\hline $\operatorname{Ln}$ (Indicador de transportes) & $\begin{array}{l}\text { Ln de km de carreteras asfaltadas y caminos } \\
\text { pavimentados }\end{array}$ & $0,048^{*}$ \\
\hline $\operatorname{Ln}($ Superficie agrícola) & Ln del número de hectáreas de superficie agrícola & $-0,069^{* * *}$ \\
\hline Ln(PEA educada) & Ln de la PEA con educación secundaria o superior & $-0,0267^{* * *}$ \\
\hline $\operatorname{Ln}($ Stock de capital privado) & $\begin{array}{l}\text { Ln del capital privado construido con datos nacionales } \\
\text { de Seminario y Beltrán (1998) y las participaciones } \\
\text { regionales de los Censos Económicos de } 1974 \text { y } 1994\end{array}$ & $0,283^{*}$ \\
\hline Número de observaciones & 92 & \\
\hline Estadístico F & $120,13^{* * *}$ & \\
\hline Test de Sargan & $15,30(p$-value $=0,29)$ & \\
\hline Test AR (1) & $-0,43(p-v a l u e=0,67)$ & \\
\hline Test AR (2) & $-0,66(p-$ value $=0,51)$ & \\
\hline Periodo & $1970-2000$ & \\
\hline Frecuencia & Quinquenal & \\
\hline
\end{tabular}


Urrunaga y Aparicio (2012)

Variable dependiente: Tasa de crecimiento del PBI per cápita

Variable explicativa

Constante

$\Delta \operatorname{Ln}(\mathrm{PBI})_{t-1}$

$\Delta \mathrm{Ln}$ (Indicador de infraestructura eléctrica)

$\Delta \operatorname{Ln}$ (Indicador de infraestructura de telecomunicaciones)

$\Delta \operatorname{Ln}$ (Indicador de infraestructura de transportes)

$\Delta \operatorname{Ln}($ Superficie agrícola)

$\Delta \operatorname{Ln}($ PEA educada)

$\Delta \operatorname{Ln}($ Stock de capital privado)
Descripción

Toma el valor de 1 en toda la muestra

Tasa de crecimiento del PBI per cápita en soles a precios de 1994 rezagado un periodo

Tasa de crecimiento de la potencia eléctrica instalada en megawatts/hora por habitante

Tasa de crecimiento del número de líneas telefónicas en servicio por habitante

Tasa de crecimiento de $\mathrm{km}$ de carreteras asfaltadas y caminos pavimentados dividido entre los $\mathrm{km}$ de red vial total

No incluida

Tasa de crecimiento de la PEA con educación secundaria o superior dividida entre la población

Tasa de crecimiento de la variable de stock de capital privado construida por Vásquez y Bendezú (2008)

hasta 2000, actualizada al 2009 con el Censo

Económico 2007 y datos sobre la inversión regionalb

$-0,0155$

\section{Parámetro} estimado

$-0,1299^{* * *}$

$0,0889^{* * *}$

$0,0831^{* * *}$

$0,0921^{* * *}$

693

Número de observaciones

Estadístico F

Test de Sargan

1,00

Test AR (1)

0,0006

Test AR (2)

0,4604

Periodo

1980-2009

Frecuencia

Fuente: Elaboración propia en base a Vásquez y Bendezú (2008) y Urrunaga y Aparicio (2012).

${ }^{a}$ Paneles dinámicos con variables instrumentales a la Arellano y Bond (1991).

${ }^{\mathrm{b}}$ Como el stock de capital privado incluye la inversión privada en electricidad, telecomunicaciones y transportes, Urrunaga y Aparicio (2008) proceden a "limpiar" la serie estimando por MCO una regresión donde la variable dependiente es el stock de capital privado y las explicativas son las tres variables de infraestructura mencionadas. La variable "stock de capital privado distinto a infraestructura en electricidad, telecomunicaciones y transportes" utilizada como explicativa del crecimiento del PBI regional per cápita corresponde a los residuos de esta regresión. Esta corrección no es realizada por Vásquez y Bendezú (2008).

..$=$ no reportado.

${ }^{* * *}=$ estadísticamente significativo al $1 \%{ }^{* *}=$ estadísticamente significativo al $5 \%{ }^{*}=$ estadísticamente significativo al $10 \%$. 
En cuanto a los parámetros estimados, se observa que los efectos asociados a los tres indicadores de infraestructura son positivos y estadísticamente significativos ${ }^{3}$. Según las estimaciones de Vásquez y Bendezú (2008), el impacto sobre el crecimiento es mayor en el caso de la infraestructura eléctrica donde un aumento en la potencia instalada de un megawatt/hora redundaría en un incremento en la tasa de crecimiento del PBI de 0,114 puntos porcentuales. En lo referente a la infraestructura de telecomunicaciones y de transporte, este estudio calcula parámetros de 0,051 y 0,048 , respectivamente.

De otro lado, en las estimaciones de Urrunaga y Aparicio (2012), predomina en magnitud el efecto de la infraestructura de transportes, seguido de la infraestructura eléctrica. Así, un aumento de 1 punto porcentual en la tasa de crecimiento del ratio de $\mathrm{km}$ de carreteras asfaltadas a $\mathrm{km}$ de carreteras totales incrementaría la tasa de crecimiento del PBI per cápita en 0,0921 puntos porcentuales, mientras que un cambio similar en la tasa de crecimiento de la potencia eléctrica instalada en megawatts/hora per cápita aumentaría la tasa de crecimiento del PBI por habitante en 0,0889 puntos porcentuales. El parámetro estimado para la tasa de crecimiento de las líneas telefónicas por habitante es 0,0831.

Cuando se utiliza el método de Arellano y Bond (1991), se debe aplicar el test de Sargan a fin de verificar la validez de los instrumentos utilizados. En tal sentido, solo en la estimación de Vásquez y Bendezú (2008) no se podría rechazar la hipótesis nula de validez de los instrumentos (Chi-cuadrado $(12)=15,30$; $p$-value $=0,29)$. En contraste, Urrunaga y Aparicio (2012) presentan un valor del test de Sargan igual a 1,00, aunque no hacen ningún comentario al respecto, ni presentan el correspondiente p-value de la prueba ${ }^{4}$. De otro lado, los tests de auto-correlación de los errores (pruebas $\mathrm{N}(0,1)$ ) reportados, no permiten rechazar las hipótesis nulas de inexistencia de auto-correlación de primer (AR (1) $\mathrm{N}(0,1)=-0,43 ; p$-value $=0,67)$ y de segundo orden (AR (2) $\mathrm{N}(0,1)=-0,66 ; p$-value $=0,51)$ en la estimación de Vásquez y Bendezú (2008). En el caso de Urrunaga y Aparicio (2012), permitiría rechazar la hipótesis nula de inexistencia de auto-correlación de primer orden $(\operatorname{AR}(1) \mathrm{N}(0,1)=0,0006$; $p$-value no reportado), pero no de segundo orden $(\operatorname{AR}(2) \mathrm{N}(0,1)=0,46 ; p$-value no reportado). En general, los resultados encontrados por Urrunaga y Aparicio (2012) es lo que se hubiera esperado encontrar si las perturbaciones del modelo en niveles hubieran estado no correlacionadas serialmente, habida cuenta de la transformación de primeras diferencias utilizada. Sin embargo, lo encontrado por Vásquez y Bendezú (2008) no invalida sus resultados5.

\footnotetext{
3 Aunque el de transportes solo al 10\% en el estudio de Vásquez y Bendezú (2008).

4 En favor de estos autores, debe decirse que realizan la estimación del modelo utilizando cinco diferentes métodos: pool de datos, panel estático (estimador MCO), panel estático (estimador intra-grupos), panel dinámico (estimados MGM), panel dinámico (estimador MGM en sistema). Los resultados reportados en el Cuadro 5 corresponden al cuarto método empleado siguiendo la metodología de Arellano y Bond (1991).

5 Ver Arellano et al. (2001).
} 


\section{CRECIMIENTO E INVERSIÓN ENTRANSPORTES Y COMUNICACIONES EN LAS REGIONES DEL PERÚ}

\subsection{El ENFOQUE CONVENCIONAL}

\section{El modelo}

Para explicar cómo la inversión regional en transportes y comunicaciones (T\&C) afecta al producto de cada región se parte de una función de producción que toma en cuenta como factores productivos a los valores rezagados del capital físico, el capital humano, el empleo y la inversión ejecutada en actividades y proyectos de T\&C. La inclusión de los valores rezagados de las variables se explica por dos razones. Desde el punto de vista analítico, para dar cuenta del tiempo que le toma a los factores productivos y a la inversión en devenir productivos. Desde el punto de vista metodológico, para evitar la posible endogeneidad de los factores productivos y de la inversión en $T \& C$ respecto de la tasa de crecimiento, de modo que estas variables son predeterminadas al PBI de cada periodo. Entonces, la función de producción a considerar es la siguiente:

(4) $Y_{i t}=A_{i t} \cdot K_{i t-1}^{\eta} \cdot\left(H_{i t-1} \cdot L_{i t-1}\right)^{\varphi} \cdot T_{i t-1}^{\psi} \cdot C_{i t-1}^{\omega}$

donde $Y$ es el PBI, $A$ es el parámetro de productividad o PTF, K es el stock de capital físico, $H$ es un indicador del stock de capital humano, y $L$ es el nivel de empleo. El producto de $H$ y $L$ representa la fuerza laboral ajustada por capital humano. $T$ y $C$ son la inversión pública en transportes y en comunicaciones, respectivamente. $\eta, \varphi, \psi$ y $\omega$ son los coeficientes asociados a cada uno de los factores productivos y representan las elasticidades de cada uno con respecto al producto. Estos coeficientes pueden sumar 1, menos que 1 o más que 1 , según el tipo de retornos a escala que presente la tecnología Cobb-Douglas utilizada. El subíndice $i$ representa la región y el subíndice $t$ el año.

La función de producción (4) se puede transformar de tal manera que se tenga una ecuación que exprese el producto por trabajador. En el caso de que se considere que existen rendimientos constantes a escala, se tiene:

(5) $y_{i t}=A_{i t} \cdot k_{i t-1}^{\eta} \cdot\left(H_{i t-1}\right)^{\varphi} \cdot t_{i t-1}^{\psi} \cdot c_{i t-1}^{\omega}$

Si no se consideran rendimientos constantes, la ecuación (4) en términos por trabajador será:

(6) $y_{i t}=A_{i t} \cdot K_{i t-1}^{\eta} \cdot H_{i t-1}^{\varphi} \cdot L_{i t-1}^{\varphi-1} \cdot T_{i t-1}^{\psi} \cdot C_{i t-1}^{\omega}$

Donde las variables en minúsculas representan las correspondientes en mayúsculas por trabajador. Como el producto por trabajador, $y$, se puede interpretar como la productividad laboral, podría surgir confusión con el término $A$, que es la PTF, y que en realidad captura todos los factores que afectan al PBI por trabajador, distintos de los factores productivos que aparecen en (4). 
Cabe señalar que la aparición de la inversión en transportes $(T)$ y en comunicaciones $(C)$ cumplen un rol similar al gasto público productivo $(g)$ del modelo de Barro (1990) discutido en la tercera sección, que se combina con una definición amplia de capital (que incluye capital físico y capital humano) y un parámetro de productividad equivalente a $A$.

\section{Datos}

Para realizar la estimación se construyó un panel de datos que comprende información de frecuencia anual para cada una de las 24 regiones del Perú en el periodo 2005-2014 Para la variable $Y$ se usa el valor agregado bruto de las regiones expresado en nuevos soles a precios de 2007. Dado que esta serie por región solo está disponible a partir del 2007, fue necesario convertir los datos de los ańos anteriores usando la tasa de crecimiento real a precios de 1994. La variable $H$ es el promedio de años de escolaridad de la población mayor de 15 años en cada región, mientras que $L$ corresponde al número de personas ocupadas en cada región. Todos estos datos fueron tomados de informes y bases de datos del Instituto Nacional de Estadística e Informática (INEI).

Las variables $T_{i t}$ y $C_{i t}$ representan la inversión pública total en actividades y proyectos en los sectores transportes (aéreo, ferroviario, hidroviario, terrestre y urbano) y comunicaciones, respectivamente. Esto incluye la inversión de los tres niveles de gobierno en cada región. Las series fueron obtenidas del sistema de Consulta Amigable del Ministerio de Economía y Finanzas (MEF). Dado que las series están expresadas en nuevos soles a precios corrientes, fue necesario convertirlas a nuevos soles a precios de 2007 usando un factor de inflación anual acumulada. La serie de inflación se obtuvo del Banco Central de Reserva del Perú (BCRP).

Finalmente, fue necesario construir la serie para la variable de stock de capital físico $(K)$. El primer paso fue construir una serie de stock capital físico a nivel nacional entre 1990 y 2014. El año inicial es 1990 pues es a partir de ese año en que se logra estabilizar la economía e iniciar un intenso proceso de acumulación de capital, luego del desastre económico experimentado durante los años 80 . El punto de partida es la ecuación de movimiento del stock de capital físico:

(7) $K_{t+1}=(1-\delta) \cdot K_{t}+I_{t}$

donde $I$ es el nivel de inversión y $\delta$ es la tasa de depreciación. Los datos de la primera variable se obtuvieron de la serie de inversión bruta interna en nuevos soles del 2007 elaborada por el BCRP. En cuanto a la tasa de depreciación, se asume una función de depreciación lineal y una vida útil del capital de 30 años, es decir, $\delta=3,3 \%$.

${ }^{6}$ Se considera a Lima y Callao como una única región. 
Para estimar el stock de capital inicial (en 1990), $K_{0}$, a partir del cual aplicar la ecuación de movimiento (7) se utiliza el método de inventarios perpetuos (Sosa et al., 2013), de modo que:

(8) $K_{0}=\frac{I^{*}}{(1+g)-(1-\delta)}=\frac{I^{*}}{g+\delta}$

donde $g$ es la tasa de crecimiento de largo plazo del PBI, e $I^{*}$ se calcula a partir de:

(9) $I^{*}=\left(\sum_{t=1951}^{t=1990} \frac{I_{t}}{Y_{t}}\right) \cdot Y_{1990}$

La idea detrás de (9) es que, a fin de minimizar los errores de medición del stock de capital inicial, se va lo suficientemente atrás en el tiempo (en este caso, 1951), de modo tal que para el primer año relevante en la estimación (1990) el stock de capital inicial heredado del pasado $\left(K_{1950}\right)$ se haya depreciado totalmente, de modo que $K_{1990}$ solo contenga la inversión acumulada a partir de 1951, que proviene directamente de las cuentas nacionales oficiales. Las fuentes de datos para estimar $I^{*}$ provienen del BCRP y están expresadas en nuevos soles a precios constantes de 2007. Por otro lado, $g$ es la tasa de crecimiento anual promedio del PBI en nuevos soles a precios de 2007 entre 1950 y 1990, calculada con datos del BCRP.

Una vez que se obtiene la serie de stock de capital físico a nivel nacional expresado en nuevos soles a precios de 2007, se procede a construir las correspondientes series regionales. Dado que no se cuenta con información sobre la composición del stock de capital físico por regiones, esta se debe de aproximar a partir de los datos disponibles. En este caso, se usó la información del Censo Económico de 2007 del INEI, de donde se tomó la información del activo fijo de las empresas censadas. Con esto se obtuvo el porcentaje que el activo fijo de las empresas de cada región representan en el activo fijo total nacional. Estos porcentajes se aplican a la serie del stock de capital físico a nivel nacional para obtener una serie aproximada del stock de capital físico por regiones. Finalmente, a fin de evitar la doble contabilidad del aporte de la inversión en T\&C en el crecimiento, se le resta a cada una de estas series regionales de stock de capital físico, la inversión rezagada un periodo en actividades y proyectos de T\&C.

\section{Resultados}

El objetivo es estimar los coeficientes $\eta, \phi, \psi$ y $\omega$ de las funciones de producción (4), (5) y (6). Es decir, se quiere encontrar el efecto del capital físico, del trabajo ajustado por capital humano, y de la inversión pública en transportes y en comunicaciones sobre el producto regional. En primer lugar, tomando logaritmo natural a ambos lados de las tres ecuaciones, se expresa las funciones de producción en términos lineales, donde los parámetros de interés a estimar se interpretan como elasticidades. El método de estimación 
corresponde a efectos fijos para paneles estáticos ${ }^{7}$. Se procede de esta manera puesto que la especificación es estática, al no incluir valores rezagados de la variable dependiente como variable explicativa. Como se señaló anteriormente, se incluyen valores rezagados de las variables explicativas para evitar problemas de endogeneidad entre estas y la variable dependiente. Cabe resaltar que las elasticidades estimadas solo capturan el impacto directo sobre el producto. No capturan los efectos indirectos, asociados, por ejemplo, a la reducción de costos de producción por la construcción y el mejoramiento de las carreteras, o a las mejoras de productividad de $K$ y de $L \cdot H$ generados por el uso de nuevos y mejores servicios de comunicaciones.

El Cuadro 5 presenta los resultados de las estimaciones de la ecuación (4) en logaritmos en dos escenarios. En el primero se supone retornos constantes a escala, por lo que la estimación se realiza imponiendo la restricción de que $\eta+\phi+\psi+\omega=1$. En el segundo, no se hace este supuesto, por lo que la estimación de (4) se realiza de manera irrestricta. La variable dependiente es el logaritmo natural del PBI regional.

Cuadro 5. Estimaciones de los determinantes del PBI regional, 2006-2014 (Variable dependiente: $\operatorname{Ln}(P B I)$; Método de Efectos Fijos)

\begin{tabular}{ccc}
\hline Variable explicativa & $\begin{array}{c}\text { Estimación restringida } \\
\text { (Retornos constantes a escala) }\end{array}$ & $\begin{array}{c}\text { Estimación } \\
\text { no restringida }\end{array}$ \\
\hline $\operatorname{Ln}\left(K_{i t-1}\right)$ & $0,552^{* * *}$ & $0,446^{* * *}$ \\
& $(0,055)$ & $(0,053)$ \\
$\operatorname{Ln}\left(L_{i t-1} \cdot H_{i t-1}\right)$ & $0,427^{* * *}$ & $0,117^{*}$ \\
& $(0,053)$ & $(0,068)$ \\
$\operatorname{Ln}\left(T_{i t-1}\right)$ & $0,0174^{* *}$ & $0,0510^{* * *}$ \\
& $(0,0080)$ & $(0,0090)$ \\
$\operatorname{Ln}\left(C_{i t-1}\right)$ & $0,00341^{*}$ & $0,00581^{* * *}$ \\
& $(0,00186)$ & $(0,00174)$ \\
$\operatorname{Constante}$ & $2,701^{* * *}$ & $9,614^{* * *}$ \\
& $(0,459)$ & $(1,077)$ \\
\hline Número de observaciones & 240 & 240 \\
Periodo & $2005-2014$ & $2005-2014$ \\
Frecuencia & Anual & Anual \\
\hline
\end{tabular}

Fuente: Estimaciones propias.

Nota: Errores estándar entre paréntesis.

${ }^{* * *}=$ significativo al $1 \% ;{ }^{* *}=$ significativo al $5 \%{ }^{*}=$ significativo al $10 \%$.

Se observa que tanto en la estimación restringida, como en la no restringida, todas las variables explicativas consideradas presentan elasticidades positivas y estadísticamente significativas, siendo mayores en magnitud la del stock de capital físico, seguida de la del

\footnotetext{
7 Se utiliza el método de efectos fijos para hacer comparables los resultados con los que se obtienen en la sección de estimaciones bajo el enfoque espacial donde las regresiones incorporan efectos fijos.
} 
trabajo ajustado por capital humano. Bajo retornos constantes a escala, un aumento de $1 \%$ de la inversión en transportes en una región, elevaría su PBI en 0,02\%. En este escenario, el impacto de un incremento de la inversión pública en comunicaciones sería aún menor, dado que presenta una elasticidad estimada de 0,003 . Si bien en la estimación no restringida, la suma de los coeficientes estimados es menor que 1, lo que indica retornos decrecientes a escala, aumentan las elasticidades estimadas tanto de la inversión pública en transportes como en comunicaciones: un incremento de $1 \%$ de la inversión pública en transportes (comunicaciones ${ }^{8}$ ), aumentaría el PBI regional en 0,05\% (0,006\%).

En el Cuadro 6 se presentan los resultados de las estimaciones de efectos fijos ${ }^{9}$ del PBI por trabajador en logaritmos con retornos constantes a escala y sin esta restricción. En la estimación restringida (ecuación (5)), todas las elasticidades estimadas son positivas, pero la asociada a la inversión en comunicaciones no es estadísticamente distinta de cero. En magnitud, las elasticidades del stock capital por trabajador $(k)$ y del stock de capital humano $(H$, los años de escolaridad de la población mayor a 15 años) prevalecen, sumando más de 0,97. En el caso de la elasticidad de la inversión en transportes por trabajador $(t)$, un aumento de $1 \%$ en $t$ generaría un incremento de $0,02 \%$ del PBI por trabajador.

Cuadro 6. Estimaciones de los determinantes del PBI regional por trabajador, 2006-2014 (Variable dependiente: $\operatorname{Ln}(P B I$ por trabajador); Método de Efectos Fijos)

\begin{tabular}{ccc}
\hline Variable explicativa & $\begin{array}{c}\text { Estimación restringida } \\
\text { (Retornos constantes a escala) }\end{array}$ & $\begin{array}{c}\text { Estimación } \\
\text { no restringida }\end{array}$ \\
\hline $\operatorname{Ln}\left(k_{i t-1}\right)$ & $0,528^{* * *}$ & \\
& $(0,057)$ & \\
$\operatorname{Ln}\left(H_{i t-1}\right)$ & $0,446^{* * *}$ & \\
& $(0,055)$ & \\
$\operatorname{Ln}\left(t_{i t-1}\right)$ & $0,0229^{* * *}$ & \\
& $(0,0083)$ & $7,777^{* * *}$ \\
$\operatorname{Ln}\left(c_{i t-1}\right)$ & 0,00262 & $(1,542)$ \\
& $(0,00192)$ & $0,425^{* * *}$ \\
$\operatorname{Constante}$ & $2,840^{* * *}$ & $(0,065)$ \\
& $(0,474)$ & \\
$\operatorname{Ln}\left(K_{i t-1}\right)$ & & \\
\end{tabular}

\footnotetext{
8 Se debe de resaltar que la mayor parte de la inversión en comunicaciones en el Perú es de carácter privado. De allí que el coeficiente estimado correspondiente a la inversión pública en comunicaciones resulte de baja magnitud en comparación a los demás. Esto aplica a todas las estimaciones realizadas en el presente trabajo.

$9 \mathrm{Al}$ igual que en el caso de la estimación en niveles, se elige directamente una estimación de efectos fijos por motivos de comparabilidad de los resultados con los de las estimaciones bajo el enfoque espacial.
} 


\begin{tabular}{ccc}
\hline Variable explicativa & $\begin{array}{c}\text { Estimación restringida } \\
\text { (Retornos constantes a escala) }\end{array}$ & $\begin{array}{c}\text { Estimación } \\
\text { no restringida }\end{array}$ \\
\hline $\operatorname{Ln}\left(H_{i t-1}\right)$ & $0,163^{*}$ \\
& & $(0,092)$ \\
$\operatorname{Ln}\left(L_{i t-1}\right)$ & $-0,708^{* * * a}$ \\
& $(0,162)$ \\
$\operatorname{Ln}\left(T_{i t-1}\right)$ & $0,0493^{* * *}$ \\
& & $(0,0097)$ \\
$\operatorname{Ln}\left(C_{i t-1}\right)$ & $0,00443^{* *}$ \\
& & $(0,00188)$ \\
\hline Número de observaciones & 240 & 240 \\
Periodo & $2005-2014$ & $2005-2014$ \\
Frecuencia & Anual & Anual \\
\hline
\end{tabular}

Fuente: Estimaciones propias.

Nota: Errores estándar entre paréntesis.

${ }^{* * *}=$ significativo al $1 \% ;{ }^{* *}=$ significativo al $5 \%{ }^{*}=$ significativo al $10 \%$.

${ }^{a}$ Corresponde al estimado del coeficiente $\phi-1$ de la ecuación (6).

Cuando se observan los resultados de la estimación no restringida (última columna del Cuadro 7), el valor negativo de la elasticidad estimada para el trabajo $(L)$ se debe a que esta representa el valor de $\phi-1$ en la ecuación (6). De este modo, el valor "verdadero" del coeficiente $\phi$ estaría entre 0,163 (la elasticidad estimada para el stock de capital humano, $H$ ) y 0,292 (= $1-0,708)$. En cuanto al aporte de la inversión pública en transportes, un aumento de $1 \%$ en este indicador aumentaría el PBI por trabajador en $0,05 \%$. De manera similar, un aumento de $1 \%$ en la inversión pública en comunicaciones incrementaría en $0,004 \%$ el producto por trabajador.

\subsection{El ENFOQUe ESPACIAL}

\section{Metodología}

Aparte del enfoque tradicional desarrollado en la subsección anterior, el impacto de la inversión en T\&C también puede evaluarse a partir del enfoque espacial. El punto de partida es una función de producción similar a la formulada en la ecuación (4):

$$
\text { (10) } Y_{i t}=A_{i t} \cdot K_{i t-1}^{\eta} \cdot\left(H_{i t-1} \cdot L_{i t-1}\right)^{\varphi} \cdot T_{i t-1}^{\psi} \cdot C_{i t-1}^{\omega}
$$

donde la interpretación de los factores productivos $(K, H, L, T, C)$, de las respectivas elasticidades factor-producto $(\eta, \phi, \psi, \omega)$, del coeficiente de productividad $(A)$ y del nivel de producto $(Y)$, es la misma que la presentada en la subsección anterior. Lo mismo sucede con los subíndices $i$ y $t-1$ en cada variable.

Tomando logaritmo natural en (10):

(11) $\operatorname{Ln}\left(Y_{i t}\right)=\operatorname{Ln}\left(A_{i t}\right)+\eta \cdot \operatorname{Ln}\left(K_{i t-1}\right)+\varphi \cdot \operatorname{Ln}\left(H_{i t-1} L_{i t-1}\right)+\psi \cdot \operatorname{Ln}\left(T_{i t-1}\right)+\omega \cdot \operatorname{Ln}\left(C_{i t-1}\right)$ 
Tal como se realizó en la subsección anterior, a la ecuación (11) se la puede estimar mediante el método de efectos fijos, con lo que se debería poder capturar las relaciones entre factores y producto de cada una de las regiones del país. No obstante, este enfoque convencional no espacial omite el hecho de que puede haber interacciones entre las variables de cada región (Belotti et al., 2012; Elhorst, 2014a, 2014b; Gibbons y Overman, 2012; LeSage y Pace, 2009).

Por ejemplo, en el caso que se está evaluando, un proyecto de inversión en infraestructura en transportes y comunicaciones no solo trae progreso a la región en donde se ejecuta el proyecto, sino que las regiones vecinas también podrían verse beneficiadas (o perjudicadas), vía una serie de canales, incluyendo la presencia de externalidades positivas (o negativas) que impactan directamente sobre el producto. En consecuencia, parece pertinente explorar esta posibilidad.

A diferencia de lo que sucede en el enfoque convencional, las regresiones espaciales capturan las correlaciones que surgen entre las variables en el espacio de los individuos a considerar. Con esto es posible estimar no solo los efectos directos de los cambios de una variable exógena en una región sobre la variable endógena de esa misma región, sino que permite un análisis mucho más completo, al poder estimar también la presencia de efectos indirectos que van de una región a otra (Anselin, 1988, 2010). Asimismo, el uso de regresiones espaciales permite alcanzar mejores estimadores, ya que ignorar la dimensión espacial del problema en casos en que sean potencialmente significativos puede llevar a estimadores ineficientes y sesgados (LeSage y Pace, 2009).

La dimensión espacial del problema se introduce a través del uso de las llamadas matrices espaciales, $W$. Estas matrices capturan la distancia espacial entre cada región a considerar y se representa de la siguiente forma:

(12) $W=\left[\begin{array}{cccc}0 & w_{12} & \ldots & w_{1 n} \\ w_{21} & 0 & \ldots & w_{2 n} \\ \vdots & \vdots & \ddots & \vdots \\ w_{n 1} & w_{n 2} & \ldots & 0\end{array}\right]_{n x n}$

De este modo, la matriz es de dimensión $n \times n$, donde $n$ es el número de regiones. Cada elemento $w_{i j}$ representa la distancia o la contigüidad existente entre las regiones $i$ y $j$. Asimismo, se tiene que $w_{i j}=w_{j i}$ cuando $i \neq j$ y que $w_{i i}=0$, con lo cual la matriz $W$ es simétrica con elementos de su diagonal iguales a cero.

Para determinar la conveniencia econométrica del uso de modelos espaciales, es necesario indagar sobre si existe correlación espacial entre las variables del modelo a estimar. Para esto se usa la I de Moran (Fischer y Wang, 2011), que mide la correlación espacial de una determinada variable en un periodo dado, y que se expresa de la siguiente manera:

(13) $I_{i}=\left(x_{i}-\bar{x}\right) \cdot \sum_{j \in J_{i}}^{n} w_{i j} \cdot\left(x_{j}-\bar{x}\right)^{2}$ 
En este caso, se analiza la variable $x$ para la región $i$ en función de cada una de las regiones $j$, que pertenecen al conjunto de $J$ regiones vecinas. $I_{i}$ es la I de Moran para la región $i, \bar{x}$ es el promedio entre los vecinos de la región $i$ de la variable bajo análisis, $w_{i j}$ es el elemento de la matriz de pesos espaciales que relaciona las regiones $i$ y $j$, mientras que $n$ es el número de observaciones. El indicador construido a partir de (13) presenta valores entre -1 (correlación espacial negativa), $y+1$ (correlación espacial positiva).

Luego de identificar la dependencia espacial entre las variables se pasa a evaluar la representación del modelo econométrico espacial a ser utilizado, el cual posee la siguiente estructura general:

(14) $z_{i t}=\rho \cdot W \cdot z_{i t}+x_{i t} \cdot \beta+W \cdot x_{i t} \cdot \theta+\mu_{i}+\varepsilon_{i t}+\lambda \cdot W \cdot v_{i t}$

donde los subíndices $i$ y $t$ son indicadores de región y periodo, respectivamente; $z_{i t}$ es la variable endógena, $x_{i t}$ son las variables exógenas, $\beta$ es el vector de coeficientes correspondientes a las variables exógenas, $\mu_{i}$ es el efecto fijo y $\varepsilon_{i t}$ es el término de error. Adicionalmente, al tratarse de una metodología econométrica que captura la dimensión espacial del problema, se cuenta con términos adicionales: $W \cdot z_{i t} \mathrm{y} W \cdot x_{i t}$ representan las ponderaciones (dadas por la matriz espacial $W$ ) de todas las variables endógenas y exógenas de las regiones vecinas a la región $i$ en el periodo $t$, respectivamente. De manera similar, $W \cdot v_{i t}$ representa la ponderación (dada por la matriz espacial $W$ ) del componente de error espacial $v_{i t}$. Este conjunto de variables de las regiones vecinas son conocidas como "rezagos espaciales", donde $\rho, \theta$ y $\lambda$ son los coeficientes asociados a los rezagos espaciales ponderados de las variables endógenas, exógenas y errores de las regiones vecinas, respectivamente.

Como se puede observar, la metodología considera la existencia de correlación espacial de las variables endógenas, exógenas y/o de los errores. No obstante, para pasar a la etapa de estimación previamente se debe elegir una especificación para el modelo econométrico (Belotti et al., 2013; Elhorst, 2014a; Gibbons y Overman, 2012). Las especificaciones más comunes son:

(15) Modelo espacial de Durbin(SDM) $\quad \rho \neq 0 \quad \theta \neq 0 \quad \lambda=0$

Modelo espacial autorregresivo $(S A R) \quad \rho \neq 0 \quad \theta=0 \quad \lambda=0$

Modelo de error espacial(SEM) $\quad \rho=0 \quad \theta=0 \quad \lambda \neq 0$

Lo anterior quiere decir que cada especificación depende de los coeficientes que se vayan a estimar, lo que no inhabilita la posibilidad de que un coefciiente individual estimado resulte estadísticamente no significativo. La idoneidad de cada especificación debe ser demostrada mediante el uso de pruebas estadísticas para los coeficientes asociados a los rezagos espaciales, mientras que la estimación de estos modelos se logra mediante el método de máxima verosimilitud (Elhorst, 2014a, 2014b; Fischer y Wang, 2011). 
En un modelo espacial, el cambio en una variable exógena correspondiente a una determinada región no solo genera un cambio dentro de esa región, sino que puede inducir también variaciones en las demás regiones. Así, la dimensión espacial hace que la interpretación de los coeficientes $\beta$ y $\theta$ no sea directa (Debarsy et al., 2012; Elhorst, 2014b; LeSage y Pace, 2009). Por ello, es necesario calcular indicadores adicionales. Para esto se reescribe el modelo econométrico expresado en la ecuación (14) como:

(16) $(I-\rho \cdot W) \cdot z_{i t}=x_{i t} \cdot \beta+W \cdot x_{i t} \cdot \theta+\mu_{i}+\varepsilon_{i t}+\lambda \cdot W \cdot v_{i t}$

donde $I$ es la matriz identidad de dimensión $n \times n$. Asimismo, dado que el análisis se concentra en las variables exógenas, se resume a los elementos que no lo son como $R=\mu_{i}+\varepsilon_{i t}+\lambda \cdot W \cdot v_{i t}$ para obtener:

(17) $z_{i t}=(I-\rho \cdot W)^{-1} \cdot\left(x_{i t} \cdot \beta+W \cdot x_{i t} \cdot \theta\right)+R$

Y eliminando los subíndices para simplificar:

(18) $z=(I-\rho \cdot W)^{-1} \cdot(x \cdot \beta+W \cdot x \cdot \theta)+R$

De (18) se pueden obtener las derivadas parciales de la variable endógena con respecto a $x^{k}$, que representa la $k$-ésima variable exógena de cada una de las $n$ regiones. Estas derivadas forman una matriz de dimensión $n \times n$ de la siguiente forma:

$$
\text { (19) } \frac{\partial z}{\partial x^{k}}=(I-\rho \cdot W)^{-1} \cdot\left(\beta_{k} \cdot I+\theta_{k} \cdot W\right)=S_{k}(W)
$$

donde $\beta_{k}$ y $\theta_{k}$ son los coeficientes correspondientes a la $k$-ésima variable exógena. Un aspecto importante es que en (19) se cuenta con el siguiente elemento:

(20) $(I-\rho \cdot W)^{-1}=I+\rho \cdot W+\rho^{2} \cdot W^{2}+\rho^{3} \cdot W^{3}+\ldots$

donde $\rho \cdot W$ contiene los efectos marginales de primer orden de las regiones vecinas sobre la región $i$. Luego, este efecto se amplía a $\rho^{2} \cdot W^{2}$, que contiene los efectos marginales de segundo orden. Es decir, $\rho^{2} \cdot W^{2}$ muestra el efecto que tiene la región vecina de la región vecina sobre la región $i$. De manera general, el elemento $(I-\rho \cdot W)^{-1}$ puede contener los efectos marginales entre regiones hasta el $p$-ésimo orden. Así es como las derivadas parciales muestran todas las interacciones entre las regiones, incluyendo aquellas que corresponden a las más alejadas.

A partir de las derivadas parciales presentadas en la ecuación (19), LeSage y Pace (2009) proponen indicadores para cuantificar los efectos de las variables exógenas sobre la variable endógena. Estos indicadores, evaluados en la $k$-ésima variable exógena, son los efectos totales $M_{k}(W)_{t o t}$, los efectos directos $M_{k}(W)_{d i r}$, y los efectos indirectos $M_{k}(W)_{\text {ind }}$ :

(21) $M_{k}(W)_{t o t}=n^{-1} \imath^{\prime} S_{k}(W) \mathfrak{\imath}$

(22) $M_{k}(W)_{d i r}=n^{-1} \operatorname{tr}\left(S_{k}(W)\right)$

(23) $M_{k}(W)_{i n d}=M_{k}(W)_{t o t}-M_{k}(W)_{d i r}$ 
donde $\imath$ es un vector de unos de dimensión $n x 1$. Los efectos totales se entienden como la variación promedio sobre la variable endógena de la región $i$ a causa de un aumento de una unidad en la $k$-ésima variable exógena en cada una de las $n$ regiones. Por su parte, los efectos directos expresan la variación promedio sobre la variable endógena en la región $i$ cuando hay un cambio de una unidad en la $k$-ésima variable exógena de esa misma región $i$. Finalmente, los efectos indirectos expresan la variación promedio de la variable endógena en la región $i$ cuando hay un aumento de una unidad en la $k$-ésima variable exógena en cada una de las $n-1$ regiones, distintas a la región $i$. Los efectos estimados de este modo aplican para todos los periodos de la muestra.

Entonces, en lo que concierne a las elasticidades asociadas a la inversión pública en transportes o comunicaciones, el efecto total es la suma del efecto directo e indirecto. Esto es, la suma del efecto porcentual sobre el nivel del PBI de la región $i$ ante un aumento de $1 \%$ de la inversión pública en transportes o comunicaciones en la misma región $i$ (efecto directo), más el efecto porcentual sobre el nivel del PBI de la región $i$ resultante del aumento de $1 \%$ en la inversión pública en transportes o comunicaciones en las otras 23 regiones (efecto indirecto). Por tanto, el efecto total indica en cuánto aumenta porcentualmente el nivel del PBI de la región $i$ si las 24 regiones (incluyendo a la región $i$ ) elevan su inversión pública en transportes o comunicaciones en $1 \%$.

Pero, como se supone que los efectos directo, indirecto y total son, en promedio, iguales para todas las regiones, el efecto total también se puede leer como en cuánto aumenta el nivel del PBI a nivel nacional (si el PBI de todas las regiones aumenta en 1\%, el PBI del país también subirá en 1\%) ante un aumento de 1\% de la inversión pública en transportes o comunicaciones a nivel nacional (si la inversión en todas las regiones sube en $1 \%$, la inversión pública nacional también subirá en 1\%).

\section{Datos}

Para las estimaciones se utilizó el mismo panel de datos de la subsección anterior que comprende información anual para cada una de las 24 regiones del Perú entre los años 2005 y 2014.

Para la matriz espacial $W$, la literatura generalmente considera las matrices de contigüidad y la de distancias inversas (Gibbons y Overman, 2012; LeSage y Pace, 2009). En la primera, el elemento $w_{i j}$ es igual a 1 si las regiones $i$ y $j$ son contiguas, mientras que toma el valor de cero cuando las regiones en mención no tienen una frontera común. En el caso de la matriz de distancias inversas, se tiene que $w_{i j}=1 / d_{i j}$, donde $d_{i j}$ es, por lo general, un indicador de distancia euclidiana. En este caso no se usará la matriz de distancias inversas, puesto que se trata de regiones con grandes extensiones. De este modo, una matriz de distancias inversas, donde se consideran los centroides de cada región para calcular las distancias interregionales podría generar distorsiones al ignorar efectos cruzados que se dan en las fronteras de las mismas regiones, o en puntos distantes de los centroides correspondientes. 
En el caso de la matriz de contigüidad, se considerará una versión normalizada de la misma, donde los valores de cada fila se restringen para que sumen uno, con lo cual se estandarizan los efectos sobre cada una de las regiones, sin importar el número de vecinos que esta tenga (Elhorst 2014b; Fischer y Wang, 2011). La matriz de contigüidad normalizada utilizada se presenta en el Cuadro A1 del Anexo.

\section{Resultados}

El Cuadro 7 presenta los resultados para la I de Moran para cada una de las variables a considerar en los distintos años de la muestra, así como su significancia estadística. Se puede observar que las variables de interés $\operatorname{Ln}\left(T_{i t}\right)$ y $\operatorname{Ln}\left(C_{i t}\right)$ presentan correlación espacial estadísticamente significativa en algunos años. Esto brinda indicios sobre la existencia de correlación espacial en estas variables exógenas, pero no en la variable endógena $\operatorname{Ln}\left(Y_{i t}\right)$, ni en las exógenas $\operatorname{Ln}\left(K_{i t}\right)$ y $\operatorname{Ln}\left(H_{i t} \cdot L_{i t}\right)$. Con esta justificación estadística se puede proceder a la estimación del modelo espacial.

\section{Cuadro 7. I de Moran}

\begin{tabular}{|c|c|c|c|}
\hline \multirow{2}{*}{ Año } & \multirow{2}{*}{ Variable } & \multicolumn{2}{|c|}{ Contigüidad normalizada } \\
\hline & & I & Valor-p \\
\hline \multirow{5}{*}{2004} & $\operatorname{Ln}\left(Y_{i t}\right)$ & $-0,044$ & 0,499 \\
\hline & $\operatorname{Ln}\left(K_{i t}\right)$ & $-0,190$ & 0,122 \\
\hline & $\operatorname{Ln}\left(H_{i t} \cdot L_{i t}\right)$ & $-0,118$ & 0,280 \\
\hline & $\operatorname{Ln}\left(T_{i t}\right)$ & 0,139 & 0,079 \\
\hline & $\operatorname{Ln}\left(C_{i t}\right)$ & 0,335 & 0,003 \\
\hline \multirow{5}{*}{2005} & $\operatorname{Ln}\left(Y_{i t}\right)$ & $-0,058$ & 0,453 \\
\hline & $\operatorname{Ln}\left(K_{i t}\right)$ & $-0,190$ & 0,112 \\
\hline & $\operatorname{Ln}\left(H_{i t} \cdot L_{i t}\right)$ & $-0,127$ & 0,256 \\
\hline & $\operatorname{Ln}\left(T_{i t}\right)$ & 0,228 & 0,022 \\
\hline & $\operatorname{Ln}\left(C_{i t}\right)$ & $-0,166$ & 0,094 \\
\hline \multirow{5}{*}{2006} & $\operatorname{Ln}\left(Y_{i t}\right)$ & $-0,061$ & 0,443 \\
\hline & $\operatorname{Ln}\left(K_{i t}\right)$ & $-0,191$ & 0,111 \\
\hline & $\operatorname{Ln}\left(H_{i t} \cdot L_{i t}\right)$ & $-0,125$ & 0,263 \\
\hline & $\operatorname{Ln}\left(T_{i t}\right)$ & 0,087 & 0,169 \\
\hline & $\operatorname{Ln}\left(C_{i t}\right)$ & 0,155 & 0,038 \\
\hline \multirow{5}{*}{2007} & $\operatorname{Ln}\left(Y_{i t}\right)$ & $-0,073$ & 0,407 \\
\hline & $\operatorname{Ln}\left(K_{i t}\right)$ & $-0,189$ & 0,114 \\
\hline & $\operatorname{Ln}\left(H_{i t} \cdot L_{i t}\right)$ & $-0,119$ & 0,267 \\
\hline & $\operatorname{Ln}\left(T_{i t}\right)$ & $-0,124$ & 0,268 \\
\hline & $\operatorname{Ln}\left(C_{i t}\right)$ & 0,121 & 0,050 \\
\hline \multirow{5}{*}{2008} & $\operatorname{Ln}\left(Y_{i t}\right)$ & $-0,068$ & 0,422 \\
\hline & $\operatorname{Ln}\left(K_{i t}\right)$ & $-0,190$ & 0,113 \\
\hline & $\operatorname{Ln}\left(H_{i t} \cdot L_{i t}\right)$ & $-0,119$ & 0,265 \\
\hline & $\operatorname{Ln}\left(T_{i t}\right)$ & $-0,133$ & 0,245 \\
\hline & $\operatorname{Ln}\left(C_{i t}\right)$ & 0,202 & 0,017 \\
\hline
\end{tabular}




\begin{tabular}{|c|c|c|c|}
\hline \multirow{2}{*}{ Año } & \multirow{2}{*}{ Variable } & \multicolumn{2}{|c|}{ Contigüidad normalizada } \\
\hline & & I & Valor-p \\
\hline \multirow{5}{*}{2009} & $\operatorname{Ln}\left(Y_{i t}\right)$ & $-0,080$ & 0,386 \\
\hline & $\operatorname{Ln}\left(K_{i t}\right)$ & $-0,187$ & 0,118 \\
\hline & $\operatorname{Ln}\left(H_{i t} \cdot L_{i t}\right)$ & $-0,112$ & 0,286 \\
\hline & $\operatorname{Ln}\left(T_{i t}\right)$ & $-0,063$ & 0,440 \\
\hline & $\operatorname{Ln}\left(C_{i t}\right)$ & 0,002 & 0,357 \\
\hline \multirow{5}{*}{2010} & $\operatorname{Ln}\left(Y_{i t}\right)$ & $-0,092$ & 0,347 \\
\hline & $\operatorname{Ln}\left(K_{i t}^{\prime \prime}\right)$ & $-0,185$ & 0,121 \\
\hline & $\operatorname{Ln}\left(H_{i t} \cdot L_{i t}\right)$ & $-0,117$ & 0,271 \\
\hline & $\operatorname{Ln}\left(T_{i t}\right)$ & $-0,020$ & 0,427 \\
\hline & $\operatorname{Ln}\left(C_{i t}\right)$ & $-0,162$ & 0,179 \\
\hline \multirow{5}{*}{2011} & $\operatorname{Ln}\left(Y_{i t}\right)$ & $-0,097$ & 0,333 \\
\hline & $\operatorname{Ln}\left(K_{i t}\right)$ & $-0,185$ & 0,121 \\
\hline & $\operatorname{Ln}\left(H_{i t} \cdot L_{i t}\right)$ & $-0,115$ & 0,278 \\
\hline & $\operatorname{Ln}\left(T_{i t}\right)$ & 0,005 & 0,357 \\
\hline & $\operatorname{Ln}\left(C_{i t}\right)$ & 0,086 & 0,158 \\
\hline \multirow{5}{*}{2012} & $\operatorname{Ln}\left(Y_{i t}\right)$ & $-0,087$ & 0,363 \\
\hline & $\operatorname{Ln}\left(K_{i t}\right)$ & $-0,186$ & 0,120 \\
\hline & $\operatorname{Ln}\left(H_{i t} \cdot L_{i t}\right)$ & $-0,116$ & 0,274 \\
\hline & $\operatorname{Ln}\left(T_{i t}\right)$ & $-0,055$ & 0,464 \\
\hline & $\operatorname{Ln}\left(C_{i t}\right)$ & $-0,0228$ & 0,061 \\
\hline \multirow{5}{*}{2013} & $\operatorname{Ln}\left(Y_{i t}\right)$ & $-0,094$ & 0,341 \\
\hline & $\operatorname{Ln}\left(K_{i t}\right)$ & $-0,184$ & 0,123 \\
\hline & $\operatorname{Ln}\left(H_{i t} \cdot L_{i t}\right)$ & $-0,119$ & 0,266 \\
\hline & $\operatorname{Ln}\left(T_{i t}\right)$ & $-0,094$ & 0,353 \\
\hline & $\operatorname{Ln}\left(C_{i t}\right)$ & $-0,049$ & 0,484 \\
\hline \multirow{5}{*}{2014} & $\operatorname{Ln}\left(Y_{i t}\right)$ & $-0,101$ & 0,323 \\
\hline & $\operatorname{Ln}\left(K_{i t}\right)$ & $-0,187$ & 0,118 \\
\hline & $\operatorname{Ln}\left(H_{i t} \cdot L_{i t}\right)$ & $-0,120$ & 0,264 \\
\hline & $\operatorname{Ln}\left(T_{i t}\right)$ & $-0,153$ & 0,200 \\
\hline & $\operatorname{Ln}\left(C_{i t}\right)$ & $-0,035$ & 0,453 \\
\hline
\end{tabular}

Fuente: Cálculos propios.

LeSage y Pace (2009) proponen comenzar con una estimación bajo la especificación SDM, para luego probar estadísticamente la idoneidad de las otras especificaciones mencionadas en la ecuación (15). Suponiendo la existencia de efectos fijos, la ecuación a estimar bajo la especificación SDM es:

(24) $\operatorname{Ln}\left(Y_{i t}\right)=\rho \cdot W \cdot \operatorname{Ln}\left(Y_{i t}\right)+x_{i t-1} \cdot \beta+W \cdot x_{i t-1} \cdot \theta+\mu_{i}+\varepsilon_{i t}$

donde:

(25) $x_{i t-1}=\left[\begin{array}{llll}\operatorname{Ln}\left(K_{i t-1}\right) & \operatorname{Ln}\left(H_{i t-1} \cdot L_{i t-1}\right) & \operatorname{Ln}\left(T_{i t-1}\right) & \operatorname{Ln}\left(C_{i t-1}\right)\end{array}\right]$

Antes de presentar los resultados de la estimación espacial, se debe realizar las pruebas estadísticas para descartar la especificación SDM en favor de las otras especificaciones 
posibles (ecuación (15)). En primer lugar, para comprobar la especificación SAR, se debe encontrar que todos los coeficientes $\theta$ son, de manera conjunta, estadísticamente iguales a cero (Belotti et al., 2013; Gibbons y Overman, 2012). En consecuencia, se procede a probar la siguiente hipótesis nula:

(26) $\theta_{K}=\theta_{H \cdot L}=\theta_{T}=\theta_{C}=0$

donde el subíndice indica la variable a la cual está asociado cada coeficiente. El resultado del test es:

(27) $\operatorname{Prob}>\chi^{2}=0,014$

de modo que se rechaza la hipótesis nula, con lo que se puede rechazar también la especificación SAR.

En segundo lugar, para realizar la verificación de la especificación SEM, se debe cumplir que $\theta=-\rho \cdot \beta$ (Elhorst, 2014a). De esta manera, la hipótesis nula consiste en comprobar de manera conjunta el siguiente sistema de ecuaciones:

(28) $\theta_{K}=-\rho \beta_{K}$

(29) $\theta_{H \cdot L}=-\rho \beta_{H \cdot L}$

(30) $\theta_{T}=-\rho \beta_{T}$

(31) $\theta_{C}=-\rho \beta_{C}$

Donde, como en el caso anterior, los subíndices señalan la variable a la que cada coeficiente está asociado. La prueba estadística arroja el siguiente resultado:

(32) $\operatorname{Prob}>\chi^{2}=0,006$

De modo que se rechaza la hipótesis nula, descartándose entonces la especificación SEM. En consecuencia, se confirma que la especificación SDM sería la adecuada para el análisis.

En el Cuadro 8 se presentan los resultados de las estimaciones bajo la especificación $\mathrm{SDM}^{10}$. Se observa que el coeficiente $\rho$ resulta estadísticamente no significativo, lo cual significa que se tiene una especificación SDM donde las variables endógenas de las regiones vecinas no guardan correlación espacial entre sí, tal como se vio con la I de Moran

\footnotetext{
${ }^{10}$ Como se mencionó, la regresión considera a la matriz de contigüidad normalizada. A manera de comparación, se hicieron estimaciones con la matriz de contigüidad no normalizada. No obstante, los criterios de selección usuales de información - AIC y BIC—, señalaron que se debería escoger la estimación considerando la matriz de contigüidad normalizada, lo cual fue ratificado por el mayor valor del $\mathrm{R}^{2}$. Los resultados de la estimación con la matriz de contigüidad se presentan en el Cuadro A2 del Anexo. Los valores de los criterios AIC y BIC, así como el $\mathrm{R}^{2}$ para ambas estimaciones se presentan en el Cuadro A3 del Anexo. El test de Hausman para comparar los modelos de efectos fijos y efectos aleatorios resulta en un $p$-value de 0,00 para el caso de la matriz de contigüidad y un $p$-value de 0,06 para el caso de la matriz de contigüidad normalizada, por lo que se trabaja con estimaciones de efectos fijos.
} 
(ver Cuadro 8). De otro lado, los coeficientes $\beta$ y $\theta-$ y sus correspondientes valores estimados - no tienen una interpretación directa, sino que solo sirven como insumos para la construcción de los efectos directos, indirectos y totales.

Cuadro 8. Resultados de las estimaciones SDM con efectos fijos y matriz de contigüidad normalizada

\begin{tabular}{ccccccc}
\hline & $B$ & $\theta$ & $P$ & $M(W)_{\text {dir }}$ & $M(W)_{\text {ind }}$ & $M(W)_{\text {tot }}$ \\
\hline $\operatorname{Ln}\left(K_{i t-1}\right)$ & $-0,322$ & $0,725^{* * *}$ & & $-0,308$ & $0,776^{* * *}$ & $0,468^{* * *}$ \\
& $(0,286)$ & $(0,297)$ & & $(0,237)$ & $(0,246)$ & $(0,078)$ \\
$\operatorname{Ln}\left(H_{i t-1} \cdot L_{i t-1}\right)$ & $0,117^{*}$ & $-0,125$ & & $0,119^{*}$ & $-0,108$ & 0,011 \\
& $(0,064)$ & $(0,109)$ & & $(0,072)$ & $(0,123)$ & $(0,137)$ \\
$\operatorname{Ln}\left(T_{i t-1}\right)$ & $0,024^{* *}$ & 0,022 & & $0,025^{* *}$ & 0,025 & $0,050^{* * *}$ \\
& $(0,012)$ & $(0,017)$ & & $(0,012)$ & $(0,019)$ & $(0,015)$ \\
$\operatorname{Ln}\left(C_{i t-1}\right)$ & $0,000^{* * *}$ & $0,007^{* *}$ & & 0,000 & $0,008^{* * *}$ & $0,008^{* * *}$ \\
& $(0,003)$ & $(0,003)$ & & $(0,002)$ & $(0,003)$ & $(0,002)$ \\
$W \cdot \operatorname{Ln}\left(Y_{i t}\right)$ & & & 0,127 & & & \\
& & & $(0,092)$ & & & \\
\hline
\end{tabular}

Fuente: Estimaciones propias.

Nota: Errores estándar entre paréntesis.

${ }^{* * *}=$ estadísticamente significativo al $1 \%,{ }^{* *}=$ estadísticamente significativo al $5 \%,{ }^{*}=$ estadísticamente significativo al $10 \%$.

En los efectos directos, se estima que tanto el rezago temporal del empleo ajustado por educación, como el de la inversión en transportes tienen coeficientes estadísticamente significativos y positivos. Así, un aumento de $1 \%$ en el indicador de empleo ajustado por capital humano en la región $i$ origina, en promedio, un aumento de $0,119 \%$ en el producto de esa región. De manera similar, un incremento de $1 \%$ en la inversión en transportes en la región $i$ lleva a un incremento promedio de $0,025 \%$ sobre el producto de esa región.

En cuanto a los efectos indirectos, se encuentra que tanto el capital físico como la inversión en comunicaciones generan incrementos estadísticamente significativos sobre el producto de las regiones. En particular, si el stock de capital crece en $1 \%$ en todas las regiones, excepto en la región $i$, el producto de esta experimentará un crecimiento promedio de $0,776 \%$. Del mismo modo, cuando la inversión en comunicaciones se incrementa en $1 \%$ en todas las regiones, excluyendo a la región $i$, el producto de dicha región aumenta en promedio en $0,008 \%$.

Finalmente, cuando se consideran los efectos totales, se tiene que el stock de capital, la inversión en transportes, y la inversión en comunicaciones, presentan coeficientes estimados positivos estadísticamente significativos. De este modo, incrementos en las 24 regiones de $1 \%$ en estas variables, llevan a aumentos en el producto regional con promedios de $0,468 \%, 0,050 \%$ y $0,008 \%$, respectivamente. 
Estos resultados permiten concluir que la metodología planteada brinda coeficientes estimados que se ajustan a la dimensión espacial de las variables observadas. Así, se puede examinar los efectos de cada variable por separado, y realizar recomendaciones de política más precisas. Asimismo, esta metodología hace posible identificar para cada tipo de inversión (capital físico, capital humano, transportes, comunicaciones), la conveniencia de ejecutar inversiones a nivel de cada región por separado (para estimular el producto vía los efectos directos), a nivel de las regiones en su conjunto (para incentivar el crecimiento a través de los efectos indirectos), o a nivel nacional (para incentivar el producto vía los efectos totales), según las metas de crecimiento planteadas.

\section{CONCLUSIONES}

En este estudio se realiza una estimación de la relación entre la inversión en infraestructura de transportes y de comunicaciones y el crecimiento económico sobre la base de datos de panel para las 24 regiones del Perú en el periodo 2006-2014. Para tal efecto se realizan dos estimaciones distintas, pero complementarias.

En primer lugar, se realiza una estimación mediante el método de efectos fijos, donde el producto agregado está determinado por una función de producción de tipo Cobb-Douglas que tiene como factores productivos al capital físico, al trabajo ajustado por educación, a la inversión en transportes, a la inversión en comunicaciones, y a la PTF. Esta estimación se realiza en dos escenarios: uno donde se impone la restricción de retornos constantes a escala, y otro donde la estimación se realiza de manera irrestricta (Cuadro 5).

En ambos casos, todas las elasticidades factor-producto resultan positivas y estadísticamente significativas a los niveles de confianza usuales. Sin embargo, en términos de magnitud, las elasticidades asociadas a la inversión en transportes y en infraestructura aparecen como pequeńos. En la estimación restringida, un aumento de $1 \%$ en la inversión en transportes (comunicaciones) se traducirá en un incremento de $0,0174 \%$ $(0,00341 \%)$ en el PBI regional. En la estimación no restringida — que revelan la existencia de retornos decrecientes a escala_-, aunque las elasticidades suben mucho su valor, permanecen en niveles reducidos en comparación a las elasticidades asociadas al capital físico y al trabajo ajustado por capital humano. En este caso, un aumento de $1 \%$ en la inversión en transportes o en comunicaciones, incrementará el producto regional en 0,0510\% y 0,00581\%, respectivamente.

Este mismo ejercicio se realiza en términos del producto por habitante (Cuadro 6). En el caso de la estimación restringida a retornos constantes a escala, la inversión en comunicaciones aparece como no estadísticamente distinta de cero. Las demás elasticidades presentan signos positivos y significancia estadística a los niveles de confianza usuales. Según estos resultados, un incremento de $1 \%$ en la inversión en transportes estará acompańado por un aumento de 0,0229\% en el producto regional. En cuanto a la estimación 
no restringida, los resultados nuevamente indican la presencia de retornos decrecientes a escala, con todas las elasticidades estimadas con los signos esperados y significativos estadísticamente. En este caso, un incremento de 1\% en la inversión en transportes o en comunicaciones generará un aumento del PBI regional en 0,0493\% o 0,00443\%, respectivamente.

Aparte de esta aproximación convencional a la estimación de la relación entre la infraestructura de transportes y de comunicaciones y el crecimiento económico, también se realizó una estimación espacial de este vínculo. Este enfoque permite estimar no solo los efectos directos de cambios de una variable exógena en una región (la inversión en transportes y en comunicaciones) sobre la variable endógena de la misma región (el producto regional), sino también los efectos indirectos resultantes de potenciales impactos cruzados asociados a cambios en una variable exógena en una región sobre el producto en una región diferente.

Los cálculos asociados a la I de Moran indican que las inversiones en transportes $(T)$ y en comunicaciones $(C)$ presenta correlación espacial estadísticamente significativa en algunos años (Cuadro 7). Es decir, la inversión en transportes (comunicaciones) en una región está correlacionada con la inversión en transportes (comunicaciones) de otras regiones. Esto brinda argumentos estadísticos para proceder a la estimación de la regresión espacial. Un conjunto de tests estadísticos permite descartar la validez del modelo espacial auto-regresivo (SAR) y la del modelo de error espacial (SEM), en favor de la especificación del modelo espacial de Durbin (SDM).

La estimación de la regresión espacial indica que la especificación SDM no admite correlación estadísticamente significativa entre la variable endógena (el producto) de regiones distintas. Los parámetros estimados implican un efecto directo estadísticamente significativo para la inversión en transportes, y un efecto indirecto estadísticamente distinto de cero para la inversión en comunicaciones. En el primer caso, la elasticidad estimada es de 0,025 , y en el segundo de 0,008. Para ambos tipos de inversión los correspondientes efectos totales - la suma de los efectos directos más los indirectos- resultan estadísticamente significativos al $1 \%$. Así, un incremento de $1 \%$ en la inversión pública en transportes en todas las regiones aumentaría el producto regional en $0,050 \%$ en promedio. De manera similar, un incremento de $1 \%$ en la inversión en comunicaciones en todas las regiones traería aparejado un aumento del producto regional en $0,008 \%$, en promedio.

En el Cuadro 9 se sintetizan los principales resultados. En el enfoque convencional, las elasticidades estimadas en la regresión no restringida son mayores que en la regresión donde se impone retornos constantes a escala, sobre todo en la de la inversión en infraestructura de transportes. En el enfoque espacial, es interesante observar que el efecto dominante en la inversión en transportes es el directo, mientras que en la inversión en comunicaciones domina el efecto indirecto. Esto indica que el factor de externalidades positivas es más importante en el segundo sector que en el primero. 
Cuadro 9. Elasticidades estimadas de la inversión en infraestructura de transportes y de comunicaciones con respecto al PBI regional

\begin{tabular}{cccccc}
\hline & \multicolumn{2}{c}{ Enfoque convencional } & \multicolumn{3}{c}{ Enfoque espacial } \\
\cline { 2 - 6 } & \multicolumn{2}{c}{ Retornos a escala } & Efecto \\
\hline & Retornos constantes & Regresión no restringida & Efecto directo & Efecto indirecto & Efecto total \\
\hline $\mathrm{T}$ & 0,0174 & 0,0510 & 0,025 & $0,025^{\mathrm{a}}$ & 0,050 \\
$\mathrm{C}$ & 0,0034 & 0,0058 & $0,000 \mathrm{a}$ & 0,008 & 0,008 \\
\hline
\end{tabular}

Fuente: Cuadros 6 y 8.

${ }^{a}$ Estadísticamente no significativo.

Las elasticidades estimadas para la inversión pública en transportes y en comunicaciones respecto del PBI a nivel de las regiones del Perú son en su mayoría estadísticamente significativas. No obstante, sus magnitudes son pequeñas, inferiores a $0,06 \%$ en el caso de la inversión en transportes, y de $0,01 \%$ en el caso de la inversión en comunicaciones. Estos estimados no son comparables con los resultados de otros estudios para el Perú mostrados en el Cuadro 4, pues estos utilizan indicadores físicos, no inversión, para las variables de infraestructura en transportes y comunicaciones. Pero igualmente sus elasticidades estimadas parecen pequeñas: 0,051 para transportes y 0,048 para comunicaciones en Vásquez y Bendezú (2008), y 0,0831 y 0,0921, respectivamente, en Urrunaga y Aparicio (2012) ${ }^{11}$. No se conoce estudio para el Perú a nivel de regiones que utilice la inversión pública en transportes y en comunicaciones como variables explicativas del nivel del PBI o del PBI por trabajador (o de sus tasas de crecimiento). Por tanto, este estudio podría considerarse como pionero en ese sentido.

Respecto de las elasticidades estimadas por estudios con datos de panel donde los individuos son países, en el Cuadro 3 se muestran resultados de algunos que utilizan el gasto público en transportes y comunicaciones como variable explicativa de la tasa de crecimiento. Las implicancias que se pueden derivar son mixtas. De un lado, la elasticidad de la inversión pública en transportes y comunicaciones en conjunto estimada por Easterly y Rebelo (1993) para 28 países en desarrollo en el periodo 1970-1988, es de 0,16 , bastante mayor que las estimadas para la inversión pública en transportes y en comunicaciones en este estudio. De otro lado, la misma elasticidad estimada por Devarajan et al. (1993) para 43 países en desarrollo en 1970-1990, es negativa (-0,025), lo cual resulta difícil de justificar teórica o conceptualmente.

11 Esto indica que un aumento de $1 \%$ en el número de kilómetros de carreteras asfaltadas y caminos pavimentados o en el número de líneas telefónicas en servicio se traduciría en un incremento de la tasa de crecimiento del PBI regional menor a 0,06\%, según las estimaciones de Vásquez y Bendezú (2008). De manera similar, según las estimaciones de Urrunaga y Aparicio (2012), un incremento de 1\% en la tasa de crecimiento de kilómetros de carreteras asfaltadas y caminos pavimentados (como porcentaje de los kilómetros de la red vial total) y en la tasa de crecimiento del número de líneas telefónicas en servicio por habitante, no sería acompañado por una aceleración de la tasa de crecimiento del PBI regional mayor a $0,1 \%$. 
Cabe recordar la interpretación de los resultados de la estimación con el enfoque espacial. Si, por ejemplo, Huánuco aumenta su inversión pública en transportes (comunicaciones) en $1 \%$, por efecto directo su PBI aumentaría en $0,025 \%$ (0\%). Si, por el contrario, Huánuco no varía su inversión, pero las otras 23 regiones incrementan su inversión pública en transportes (comunicaciones) en $1 \%$, por efecto indirecto el PBI de Huánuco se expandiría en $0,025 \%(0,008 \%)$. Si ambos aumentos en la inversión se dan simultáneamente, por efecto total el producto de Huánuco aumentaría en $0,05 \%$ (0,008\%). Lo mismo sucederá en las otras 23 regiones, que verían su PBI aumentar por el aumento de $1 \%$ en su propia inversión pública por efecto directo, y por el incremento de $1 \%$ en la inversión de las otras 23 regiones por efecto indirecto. Pero esto implica un aumento de $1 \%$ de la inversión pública nacional en transportes (comunicaciones), lo que generaría un incremento de $0,05 \%$ $(0,008 \%)$ en cada una de las 24 regiones y a nivel nacional. Si cada región aumenta su inversión pública en $1 \%$, la inversión pública total nacional aumentará en $1 \%$, Si cada región crece en $0,05 \%(0,008 \%)$, el PBI nacional también crecerá en 0,05\%. (0,008\%).

Si bien estos efectos pueden parecer mínimos, se debe de resaltar que las estimaciones consideran solo un efecto de corto plazo de la inversión en transportes y comunicaciones sobre el PBI regional (en específico, con un rezago de un año), pero muchas veces la inversión pública en infraestructura tarda varios años en hacer sentir su efecto sobre el producto. Por tanto, el efecto de mediano y largo plazo de la inversión pública en transportes y comunicaciones sobre el PBI regional sería bastante mayor que los estimados en este trabajo. Asimismo, se debe considerar que las obras de infraestructura traen beneficios para la población, más allá del PBI. Inversiones que generan mejores carreteras, puentes, puertos, aeropuertos, ferrocarriles, y mejores servicios de telefonía fija y móvil, acceso a banda ancha de internet, entre otros, redundarán en una mejor calidad de vida en cada una de las regiones. En ese sentido, no se puede menospreciar el impacto que la inversión en transportes y comunicaciones puede tener sobre la calidad de vida de la población y sobre el PBI en el mediano plazo y en el largo plazo solo porque esta inversión presenta una baja elasticidad respecto al PBI regional en el corto plazo.

Finalmente, los resultados de las estimaciones realizadas sugieren que las políticas económicas respecto de la inversión pública en transportes y comunicaciones deberían buscar centrarse en un incremento general a nivel nacional, de todas las regiones del país. Esto es particularmente relevante para la inversión en comunicaciones, dado que (casi) todo el impacto sería indirecto, de acuerdo a las estimaciones a partir del enfoque espacial. Eso maximizaría su impacto sobre el PBI nacional y el de cada región. Por supuesto, esto siempre estará sujeto a la restricción presupuestaria del sector público.

En contextos de estrechez del presupuesto público, debería priorizarse la inversión pública en transportes por sobre la de comunicaciones, la que podría realizarse en regiones específicas, y no necesariamente a nivel nacional. Así, se incrementaría el producto en estas regiones de manera directa, y se estimularía la expansión del PBI nacional dependiendo de la participación que esas regiones tengan en el producto del país. 


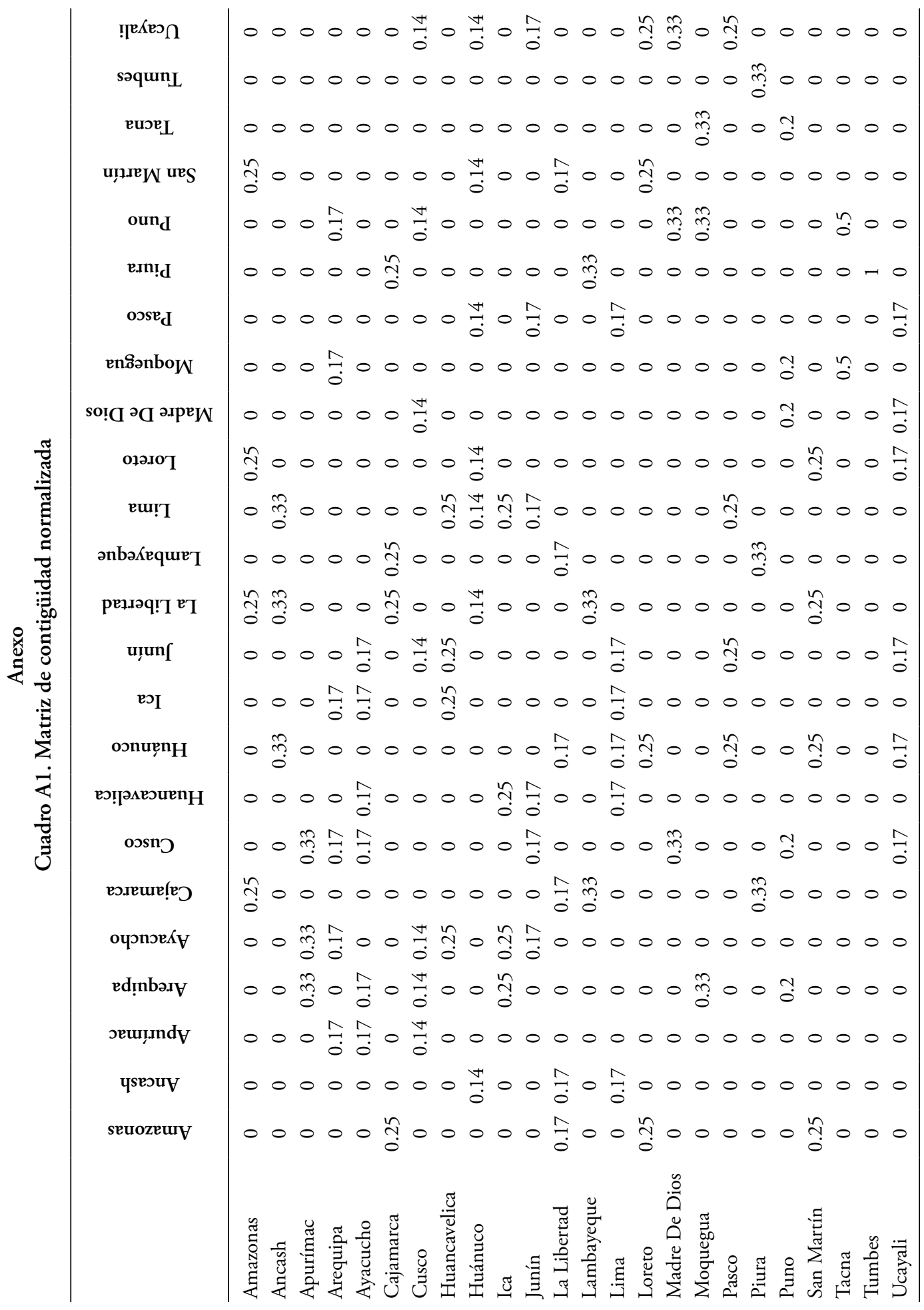


Cuadro A2. Resultados de las estimaciones SDM con efectos fijos y matriz de contigüidad

\begin{tabular}{lcccccc}
\hline & $B$ & $\theta$ & $\rho$ & $M(W)_{\text {dir }}$ & $M(W)_{\text {ind }}$ & $M(W)_{\text {tot }}$ \\
\hline $\operatorname{Ln}\left(K_{i t-1}\right)$ & 0.086 & $-0.039^{*}$ & & 0.095 & 0.100 & 0.195 \\
& $(0.086)$ & $(0.022)$ & & $(0.069)$ & $(0.135)$ & $(0.137)$ \\
\hline $\operatorname{Ln}\left(H_{i t-1} \cdot L_{i t-1}\right)$ & 0.077 & $-0.103^{* * *}$ & & $0.155^{*}$ & $0.641^{* * *}$ & $0.796^{* * *}$ \\
& $(0.067)$ & $(0.026)$ & & $(0.083)$ & $(0.245)$ & $(0.256)$ \\
\hline $\operatorname{Ln}\left(T_{i t-1}\right)$ & $0.022^{* *}$ & -0.002 & & 0.019 & -0.032 & -0.013 \\
& $(0.010)$ & $(0.004)$ & & $(0.012)$ & $(0.028)$ & $(0.033)$ \\
\hline $\operatorname{Ln}\left(C_{i t-1}\right)$ & -0.001 & 0.000 & & -0.000 & 0.001 & 0.001 \\
& $(0.002)$ & $(0.001)$ & & $(0.002)$ & $(0.004)$ & $(0.004)$ \\
\hline$W \cdot \operatorname{Ln}\left(Y_{i t}\right)$ & & & $0.277^{* * *}$ & & & \\
& & & $(0.008)$ & & & \\
\hline
\end{tabular}

Fuente: Estimaciones propias.

Nota: Errores estándar entre paréntesis.

${ }^{* * *}=$ estadísticamente significativo al $1 \%,{ }^{* *}=$ estadísticamente significativo al $5 \%,{ }^{*}=$ estadísticamente significativo al $10 \%$.

Cuadro A3. Comparación de criterios

\begin{tabular}{lcc}
\hline & Contigüidad & Contigüidad normalizada \\
\hline AIC & $-470,24$ & $-550,0$ \\
BIC & $-435,4$ & $-515,2$ \\
$\mathrm{R}^{2}$ within & 0,63 & 0,79 \\
$\mathrm{R}^{2}$ between & 0,02 & 0,39 \\
$\mathrm{R}^{2} 2$ overall & 0,02 & 0,30 \\
$p$-value de test de Hausman & 0,00 & 0,06 \\
\hline
\end{tabular}

Fuente: Estimaciones propias. 


\section{REFERENCIAS}

AFIN (2012). Por un Perú integrado: Plan Nacional de Infraestructura 2012-2021. Lima: Asociación para el Fomento de la Infraestructura Nacional.

AFIN (2015). Un plan para salir de la pobreza: Plan Nacional de Infraestructura 2016-2025. Lima: Asociación para el Fomento de la Infraestructura Nacional.

Almeida, E. y P. Guimarậes (2014). Economic growth and infrastructure in Brazil: A spatial multilevel approach. ERSA Conference Papers No. 14. European Regional Science Association.

Anselin, L. (1988). Spatial econometrics: methods and models. Berlín: Springer.

Anselin, L. (2010). Thirty years of spatial econometrics. Papers in Regional Science, 89(1), 3-25.

Arellano, M. y S. Bond (1991). Some tests of specification for panel data: Monte Carlo evidence and an application to employment equations. Review of Economic Studies, 52(2), 277-297.

Arellano, M., S. Bond, J. A. Doornik, y D. F. Hendry (2001). Panel data models. En J. A. Doornik y D. F. Hendry (eds.), Econometric modelling using PcGive, vol. 3. Londres.

Aschauer, D. A. (1989). Is public expenditure productive? Journal of Monetary Economics, 23(2), 177-200.

Barro, R. (1990). Government Spending in a Simple Model of Endogeneous Growth. Journal of Political Economy, 98(5), S103-126.

Belotti, F., G. Hughes y A.P. Mortari (2013). XSMLE-A Command to estimate spatial panel models in Stata. German Stata Users Group Meeting.

Calderón, C. y L. Servén (2004). The effects of infrastructure development on growth and income distribution. Policy Research Working Paper 3400. Washington, DC: Banco Mundial.

Calderón, C. y L. Servén (2014). Infrastruture, growth, and inequality. Policy Research Working Paper 7034. Washington, DC: Banco Mundial.

Canning, D. (1999). Infrastructure's contribution to aggregate output. Policy Research Working Paper 2246. Washington, DC: Banco Mundial.

Debarsy, N., C. Ertur, y J.P. LeSage (2012). Interpreting dynamic space-time panel data models. Statistical Methodology, 9(1), 158-171.

Devarajan, S., V. Swaroop y H. Zou (1996). The composition of public expenditure and economic growth. Journal of Monetary Economics, 37(2), 313-344.

Dugall, V., C. Saltzman, y L. Klein (1999). Infraestructure and productivity: A nonlinear approach. Journal of Econometrics, 92(1), 47-74.

Easterly W. y S. Rebelo (1983). Fiscal policy and economic growth: An empirical investigation. Journal of Monetary Economics, 32(4), 417-458.

Elhorst, J. P. (2014a). Spatial econometrics: from cross-sectional data to spatial panels. Berlín: Springer.

Elhorst, J. P. (2014b). Spatial panel models. En M. M. Fischer y P. Nijkamp, Handbook of Regional Science. 1637-1652. Berlín: Springer.

Esfahani, H. y M. Ramírez (2003). Institutions, infrastructure, and economic growth. Journal of Development Economics, 70(2), 443-477.

Fischer, M. M. y J. Wang (2011). Spatial data analysis: models, methods and techniques. Berlín: Springer Science \& Business Media.

Futagami, K., Y. Morita y A. Shibata (1993). Dynamic analysis of an endogenous growth model with public capital. Scandinavian Journal of Economics, 95(4), 607-625.

García-Milà, T., T. McGuire y R. Porter (1996). The effect of public capital in state-level production functions reconsidered. Review of Economics and Statistics, 78(1), 177-180. 
Gibbons, S. y H.G. Overman (2012). Mostly pointless spatial econometrics? Journal of Regional Science, 52(2), 172-191.

Gramlich, E. M. (1994). Infrastructure investment: A review essay. Journal of Economic Literature, 32(3), 1176-1196.

LeSage, J. P. y R.K. Pace (2009). Introduction to spatial econometrics. Londres: Chapman \&Hall/ CRC.

Munnell, A. H. (1990). How does public infrastructure affect regional economic performance? New England Economic Review, setiembre, 11-33.

Pritchett, L. (2000). The tyranny of concepts: CUDIE (Cumulated, Depreciated, Investment Effort) in not capital. Journal of Economic Growth, 5(4), 361-384.

Rivera, J. y P. Toledo (2004). Efectos de la infraestructura pública sobre el crecimiento de la economía, evidencia para Chile. Estudios de Economía, 31(1), 21-38.

Rosenstein-Rodan, P.N. (1943). Problems of industrialization of Eastern and South-Eastern Europe. Economic Journal, 53, 202-211.

Sánchez-Robles, C. (1998). Infrastructure investment and growth: Some empirical evidence. Contemporary Economic Policy, 16(1), 98-108.

Seminario, B. y A. Beltrán (1998). Crecimiento económico en el Perú 1896-1995: Nuevas evidencias estadisticas. Documento de Trabajo 32. Lima: Universidad del Pacífico.

Servén, L. (2015). Infrastructure and economic development. Presentación en la Reunión Anual del Fondo Monetario Internacional y el Banco Mundial. Lima. Mimeo.

Sosa, S., E. Tsounta y H.S. Kim (2013). Is the Growth Momentum in Latin America Sustainable? Working Paper No. 13/109. Washington, DC: Fondo Monetario Internacional.

Straub, S. (2011). Infrastructure and development: A critical appraisal of the macro-level literature. Journal of Development Studies, 47(5), 683-708.

Straub, S., C. Vellutini y M. Walters (2008). Infrastructure and economic growth in East Asia. Policy Research Working Paper No. 4589. Washington, DC: Banco Mundial.

Urrunaga, R. y C. Aparicio (2012). Infraestructura y crecimiento en el Perú. Revista de la CEPAL, 107, 157-177.

Vásquez, A. y L. Bendezú (2008). Ensayos sobre el rol de la infraestructura vial en el crecimiento económico del Perú. Lima: Consorcio de Investigación Económica y Social (CIES) y Banco Central de Reserva del Perú (BCRP).

Webb, R. (2013). Conexión y despegue rural. Lima: Universidad San Martín de Porres e Instituto Perú. 